\title{
MODIFIED TECHNIQUE FOR MEASURING DIELECTRIC CONSTANTS \\ USING A RECTANGULAR CAVITY RESONATOR \\ by
}

J. K. Sinha

Electrical Engineering Department.

Rice University

Houston, Texas

SUMMARY

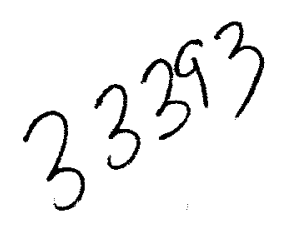

A modified technique using a partially filled rectangular cavityresonator is described. This technique is analogous to the technique described earlier by the author in which a partially filled tunable cylindrical cavity resonator is employed. The theory and the method of operation of this technique are discussed and the special advantages resulting from its use are pointed out.
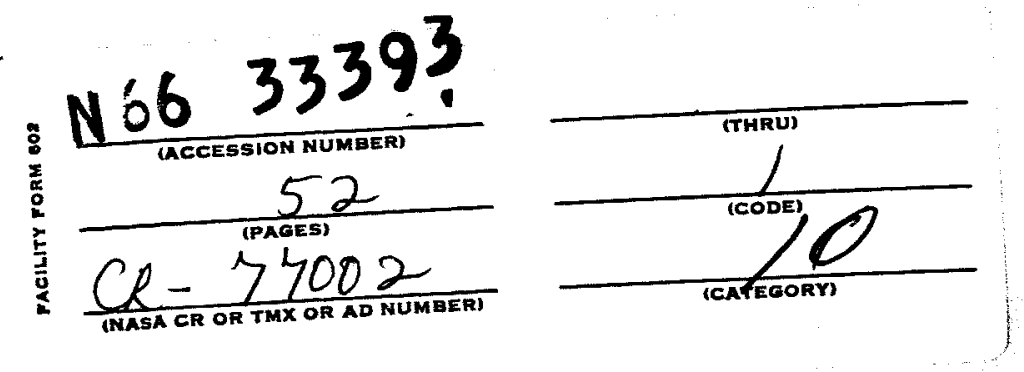


\section{Introduction}

A number of resonance methods of dielectric measurements have been described earlier by Lamb ${ }^{1}$, Horner ${ }^{2}$, Collie et $\mathrm{al}^{3}$ and Sinha ${ }^{4}$. In Lamb's method one needs a disc sample with an accurate fit with the diameter of the Ho ${ }_{\text {In }}$ cylindrical cavity resonator used. Hotston, 5 , however, has worked out a perturbation relation by which one may evaluate the real part of the permittivity in the Lamb's technique for disc samples having a considerable clearance between themselves and the inner walls of the cavity. He does not, however, investigate the possibility of the evaluation of loss-tangents for such samples and hence one needs to use a sample with a good fit for reliable evaluation of loss-tangent. Horner uses rod samples longitudinally filling a non-tunable $E_{010}$ cavity while Collie uses a thin capillary filled with water extending coaxially along the entire length of a Ho $1 \mathrm{n}$ cavity for the evaluation of the dielectric properties of water. Sinha ${ }^{4}$ and Brown, however, have described a technique using a $\mathrm{Ho}_{1 n}$ cylindrical cavity where the samples are in the form of rods which partially fill the cavity along its axis. This technique offers the facility that the sample need not have an accurate fit with respect to any one dimension of the cavity and also the cavity being tunable, all the measurements could be made at any fixed frequency.

In practice this method proved very successful except that the advantages obtained resulted from an increased computational work due to the complex solution of the Maxwell's fields inside the system and also that one is limited only to rod samples. 
As it is quite often the case that the dielectric materials are available in the form of plane sheets, it was felt desirable therefore, to have a technique which could have most of the advantages of the aforementioned technique 4 and could employ a sample in the form of a sheet or plane slab for the derivation of its electrical properties. Hence, a partially filled technique has been developed using a rectangular cavity resonator formed by using a standard $\mathrm{x}$-band waveguide, in which the samples would be in the form of thin sheets partially filled the cavity along its length and having their widths equal to the narrow dimension of the guide. The sample could either be in contact with the sidewall or alternatively be in the center of the - cross-section of the guide. The other end of the guide is closed by a tuning plunger. The cavity is coupled to the generator and the detector through holes in the side walls.

It must be mentioned here, hovever, that although one of the major advantages available by using the cylindrical cavity resonator method, i.e., of having specimens that do not need to have an accurate fit with any of the cavity dimensions, is not present, it has all the other desirable features. In fact, as the solution of the Maxwe11's equations for this system turns out to be simpler than the solution for the cylindrical resonator system, the expressions for the various parameters in terms of the measurable quantities (the guide wavelength in the partially filled portion and the Q factors for two different insertions of the sample differing by half a wavelength) turn out to be much simpler, thereby, reducing the computational labor considerably as compared to the technique described previously ${ }^{4}$. In addition, as the cavity in this case is operated in its dominant mode and 
the dimensions of the cavity are such that theproblems due to the presence of unwanted modes are not encountered. This is a definite advantage over the author's previous technique in which the experiment is done in the HO${ }_{1 n}$ mode which is not the dominant mode of the cylindrical cavity. Hence, special precautions are taken in dimensioning the cavity for the suppression of the unwanted modes.

It is also believed that the limitation of having the sample width equal to the narrow dimension of the cavity is not a great inconvenience due to the simple shape of the sample and the convenient size of the cavity's cross section.

In addition, the samples can either be in the center where the electric field and hence the perturbation is the maximum or alternately the sample could be at the side walls when the perturbation is small. One can use the former configuration for the investigations of low permittivity and 1 ow electric loss materials while the latter configuration will offer advantages for the materials having high permittivities and higher electrical losses. Outline of the Method

The procedure involved in the evaluation of the various parameters are exactly the same as in the author's previous method and is outlined below.

The real part of the permittivity is evaluated from the measured value of the wavelength inside the partially filled portion of the cavity and the loss-tangenet is obtained by measuring the $Q$ of the cavity corresponding to the two insertions of the dielectric specimen differing by half a guide wavelength. 
The photograph of the cavity system used in the present work is shown in Fig.. 1. The cavity is made from a length of standard X-band waveguide and is resonant in its dominant $\mathrm{HO}_{14}$ mode. At one of the closed ends there is a provision for the tuning of the cavity by the accurately controlled movement of theplunger while at the other end provision has beenl wade for the controlled insertion of the dielectric sample inside the cavity either along its center or along its sidewall. This end of the cavity is terminated by a thick backing plate having recesses cut both at the center and along the ends. Provision has been made for closing either the central recess or the end recess on this plate depending on whether one is inserting the sample through the center or through the side. The width of these recesses can also be varied by screwing in rectangular plates in order to be able to investigate samples having different thicknesses. The other end of the sample is held by a screw in a recess on another fixed plate which is attached to the shaft of a micrometer in order to insert the sample inside the cavity by measured amounts. The backing plate on the dielectric side is chosen sufficiently thick so that no power may leak out of the cavity through the recesses provided for the insertion of the samples. The coupling to the generator and the detector is made through holes cut in the sidewalls.

The wavelength in the partially filled portion is evaluated from the curve obtained by plotting the total length of the cavity system against the length of insertion of the dielectric and the resulting curve is identical in form to those obtained by the well known Feenberg ${ }^{6}$ method.

The quality factor is evaluated at a fixed frequency from the resonance curve by detuning the cavity with the help of the tuning plunger. The 
expression correlating the half width frequency $\Delta f$ with the corresponding . $\Delta l$ is obtained by differentiating the equation as shown in the appendix. Theory

The cavity system under discussion is basically as shown in Fig. 2 consisting of a length "d" of the empty waveguide and a length "l!" of the partially filled waveguide. The junction between the two waveguides may be assumed to be loss-free and as such can be represented by an ideal transformer with a turn ration " $n$ " at certain characteristic reference planes ${ }^{7}$. The equivalent circuit of the system can, therefore, be shown as in Fig. 3 where $d_{0}$ and $l_{0}$ are taken to be the distances of the reference planes from the actual junction in order to account for the phase changes introduced. The condition that the circuit of Fig. 3 should resonate at a given free space wavelength is:

$$
\tan \beta_{1}\left(d+d_{0}\right)=-n^{2} z_{2} / z_{1} \tan \beta_{2}\left(l+l_{0}\right)
$$

- where $B_{1}, \beta_{2}$ are the phase change co-efficients and $z_{1}, z_{2}$ the wave impedances in the empty and partially filled portions of the cavity respectively. Now, instead of specifying both the impedances in the above equation one can specify only one impedance, say $z_{1}$ and put $n=1$. The value of $z_{2} / z_{1}$ is fixed. Hence $z_{2} / z_{1}$ can be used as the third parameter to give the value of the reflection coefficient at a discontinuity. Such a procedure has been justified by Collin and Brown ${ }^{8}$ by using Schwinger's variational method and also by $\operatorname{sinha}^{9}$ by using a different approach. The equation (1), therefore, is now written as:

$$
\tan \beta_{1}\left(d+d_{0}\right)=-z_{2} / z_{1} \tan \beta_{2}\left(l_{l} l_{0}\right)
$$


or

$$
\tan \beta_{1} d^{\prime}=z_{2 / z_{1}} \tan \beta_{2} l^{\prime} \cdots \cdots
$$

where

$$
d^{\prime}=d+d_{0} \text { and } l^{\prime}=l+l_{0}
$$

The above equation coupled with Feenberg's theory can therefore be used to predict the form of dependence between $l^{\prime}$ and $d^{\prime}$. In the present application it is most convenient to plot $\left(d^{\prime}+\ell^{\prime}\right)$ against $\ell^{\prime}$. The curve of $\left(d^{\prime}+l^{\prime}\right)$ against $l^{\prime}$ has the shape shown in Fig. 4. The curve of $(d+\ell)$ vs $\ell$ will also have the same slope but will be displaced by an amount $l_{0}$ along the horizontal axis and by an amount $\left(d_{0}+l_{0}\right)$ along the vertical axis. The man slope of the curve, i.e., the slope of the dotted line, is given by $\frac{\left(\beta_{2}-\beta_{1}\right)}{\beta_{2}}$. The value of this slope can be measured from experiment and $\beta_{1}$ can be simply calculated. This enables the determination of $\beta_{2}$, i.e., the phase change coefficient in the partially filled portion. Hence, the only remaining step in the deteraination of $\epsilon_{r}$ is the correlation of $\beta_{2}$ with $\epsilon_{r}$.

It may also be mentioned that the values of $d_{0}$ and $l_{0}$, defined in Eq. (3), can be determined from the curve of $(d+l)$ vs $\ell$ as it can be shown that corresponding to its point of maximum slope,

$$
d+l+d_{0}+l_{0}=p \lambda_{1 / 4}+q \lambda_{2 / 4} \ldots
$$

and

$$
l+l_{0}=q \lambda_{2 / 4} \ldots \ldots \ldots
$$

$\mathbf{p}$ and $\mathbf{q}$ in the above equations are integers and easily deducible from the known values of $\lambda_{1}, \lambda_{2}$ and the physical length of the cavity. The values of $d_{0}$ and $l_{0}$ are needed in some of the derivations as will be seen later. 
The foregoing discussion is valid for both the cases, i.e., when the sample is in contact with the side wall and also when the sample is at the center of the cross section.

Evaluation of $\varepsilon_{2}$ from $\mathrm{B}_{2}\left(\lambda_{2}\right)$

(i) Gase when the sampie is in contact with the sidewall

The arrangement for this case is shown in Fig. 5. Pincherley ${ }^{10}$ has considered such a system and obtained the characteristic equations for the $\mathrm{HO}_{1}$ mode supporting $\mathrm{E}_{\mathrm{x}}, \mathrm{H}_{\mathrm{y}}$ and $\mathrm{H}_{\mathrm{z}}$. The same equation, however, is more readily obtained from transverse resonance conditions and is written as

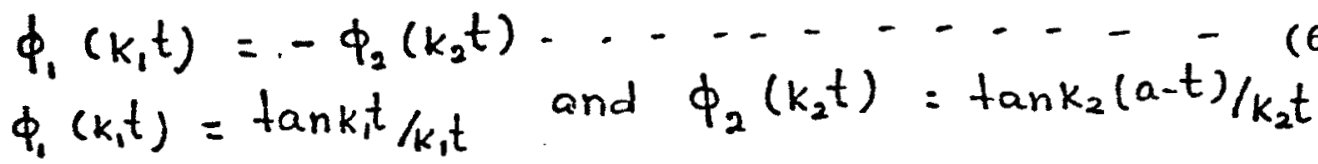

$K_{1}$ and $K_{2}$ appearing in the above equations are of the nature of propagation constants and are given by:

$$
\begin{aligned}
& k_{1}^{2}=\omega^{2} \mu_{0} \varepsilon_{1}-\beta^{2}-\cdots- \\
& k_{2}^{2}=\omega^{2} \mu_{0} \varepsilon_{0}-\beta^{2} \cdots \ldots \ldots \ldots
\end{aligned}
$$

where $\epsilon_{1}$ is the permittivity of the sample and $\beta$ is the phase change coefficient inside the guide system.

The procedure for the. evaluation of $\epsilon_{r}$ from $\beta$ is as follows. $K_{2}$ is first obtained from Eq. ( 8 ) and $K_{1}$ may then be evaluated from the transcedental Eq. (6). The substitution of $K_{1}$ in the Eq. (7) now enables the determination of $\varepsilon_{1}$ or $\epsilon_{1} / \varepsilon_{0}$, the relative permittivity of the sample.

In practice, however, it is desirable to have charts from which the dielectric parameters could be read directly, given the required experimental data. Such charts have been computed for a range of the sample thicknesses and for three different frequencies, i.e., $8 \mathrm{Kmc}, 10 \mathrm{Kmc}$ and $12 \mathrm{Kmc}$, and 
are given in Figs. 6,7 and 8 respectively.

(ii) The sample along the center of the cross section of the guide.

The cross section of the arrangement having the sample along the center of the guide is shown in Fig. (9). Such a system has been considered earlier by Vartanian ${ }^{12}$ et al, who obtained the characteristic equation for this system; supporting either $\mathrm{E}$ or $\mathrm{H}$ mode. However, the same equation is obtained simply by the transverse resonance conditions and is given for the dominant $\mathrm{HO}_{1}$ mode as

$$
\phi_{1}^{\prime}\left(k_{1}^{\prime} t^{\prime}\right)=2 d / t \cdot \phi_{2}^{\prime}\left(k_{2}^{\prime} d\right) \cdots \ldots
$$

where

$$
\phi_{1}^{\prime}\left(k_{1}^{\prime} t^{\prime}\right)=\frac{\cot k_{1}^{\prime} t^{\prime} / 2}{k_{1}^{\prime} t^{\prime} / 2} \text { and } \phi_{2}^{\prime}\left(k_{2}^{\prime} d\right)=\tan k_{2}^{\prime} d / k_{2}^{\prime} d
$$

Once again, $K_{1}^{\prime}$ and $K_{2}^{\prime}$ in Eq. (9) are given by the following relations:

$$
\begin{aligned}
& k_{1}^{\prime 2}=\omega_{0}^{\prime 2} \mu_{0} \varepsilon_{1}-\beta^{\prime 2} \cdots \cdots \\
& k_{2}^{\prime 2}=\omega_{0}^{\prime 2} \mu_{0} \varepsilon_{0}-\beta^{\prime 2} \cdots \cdots \cdots
\end{aligned}
$$

$\beta^{*}$ being the phase change coefficient for this configuration.

The procedure for evaluating $\epsilon_{\mathrm{r}}$-from Eqs. (9-11) are the same as for the previous case. The theoretical charts, for directly reading $\epsilon_{\mathbf{r}}$ from the experimental data, have been prepared for a range of sample sizes for this case and are shown in Figs. (10-12) for the respective frequencies of 8,10 and $12 \mathrm{kmc} / \mathrm{s}$.

\section{Limitation on the Thickness of the Sample}

It must be mentioned at this stage that although there is no specification regarding the precise value of the thickness to be measured by either of these methods, a Iimitation to the thickness of the sample is imposed by the condition 
of single mode propagation because above a certain value of the thickness, the cavity in addition to the dominant mode can support the next higher mode and the experimental determination of wavelength is complicated as the curve of $d+l$ vs $l$ becomes very irregular for such a case. Hence, the thickness of the sample corresponding to any particular $e_{r}$ should be such that the $\mathrm{HO}_{2}$ mode is cut off.

This value of $\epsilon_{r}$ for $\mathrm{HO}_{2}$ cut-off is determined from Eqs. (6-8) for the side filled case by putting $\beta$ equal to zero in the eqs. 7 and 8 , as the form of the characteristic equation remains unchanged for the $\mathrm{HO}_{2}$ mode. 10

For the case of the centrally filled system, however, one cannot use Dq. (9) as the characteristic equation for $\mathrm{HO}_{2}$ mode is given by 11,12

$$
\frac{\tan k_{1}^{\prime} t^{\prime} / 2}{k_{1}^{\prime}}=-\tan k_{2}^{\prime} d^{\prime} / k_{2}^{\prime} \ldots
$$

Now, by using Eqs. (10-12) and putting $\beta^{\prime}$ equal to zero, one can evaluate the maximum value of $E_{x}$ corresponding to any thickness for keeping the $\mathrm{HO}_{2}$ mode at cutoff.

It must be noted, in addition, that $K_{2}$ and $K_{2}^{\prime}$ in Eqs. (8) and (11) become imaginary if $\beta$ or $\beta^{\prime}$ exceeds $\beta_{0}$ or $\beta_{0}^{\prime}$, or in other words, the guide wavelength becomes smaller than the free space wavelength. Hence there is a limiting value of the thickness of the sample for any particular $\epsilon_{\mathbf{r}}$ beyond which one needs to evaluate the hyperbolic functions for the evaluation of the dielectric parameters. This limiting value of $\epsilon_{r}$ for any thickness for the two cases may be obtained from Eqs. (6-8) and Eqs. (9-11) by putting $\beta=\beta_{0}$ and $\beta^{\prime}=\beta_{0}^{\prime}$ into them respectively.

The curves showing the maximun value of $\epsilon_{\mathrm{r}}$ vs. thickness for the $\mathrm{HO}_{2}$ 
cutoff and for the use of only real functions for the side and centrally loaded cases are shown in Fig. (13) and (14) respectively. The Use of Hyperbolic Functions

It can be seen from Fig. (13) and (14) that the condition for $\mathrm{K}_{2}$ to be real gives a lower limiting value of $\bar{e}_{T}$ correspondinô to any thickness as compared to the value obtained for the $\mathrm{HO}_{2}$ cutoff. Hence, it is seen that in the regions between the two limiting curves, one can get single mode propagation but an imaginary value of $K_{2}^{\prime}$. Hence it is interesting to solve for the hyperbolic functions in this region as the $(d+\ell)$ vs $\ell$ curve is undistorted and enables the accurate evaluation of the guide wavelength.

Considex first the side-filled configuration. $K_{2}$ in Eq. (8) now becomes imaginary, say equal to $j \mathrm{~K}_{2}$. Hence, in the Eq. (6) $\Phi_{2}\left(\mathrm{~K}_{2} t\right)$ becomes equal to $\frac{\tan h \mathrm{~K}_{2} t}{\mathrm{~K}_{2} \mathrm{t}}$ while $\bar{\Phi}_{1}\left(\mathrm{~K}_{1} \mathrm{t}\right)$ remains unchanged, as $\mathrm{K}_{1}$ remains real due to the large values of $\epsilon_{r}$ involved. The procedure for evaluating $\varepsilon_{r}$, however, remains unaltered except for the evaluation of tanh $k_{2} t$ in place of $\tan k_{2} t$. Similarly, for the centrally loaded configuration $K_{2}^{\prime}$ in Eq. (11) turns imaginary and $\gamma_{2}^{\prime}\left(K_{2}^{\prime} d\right)$ becomes equal to $\frac{\tanh K_{2}^{\prime} d}{K_{2}^{\prime} d}$ while ${ }_{1}^{\prime}\left(K_{1}^{\prime} t\right)$ remains unaltered.

There is no theoretical limitation for not using these hyperbolic relations for the regions of the higher $H$ mode propagations. The limitation is imposed, however, from the difficulty in obtaining an undistorted experimental $(d+l)$ vs $l$ curve corresponding to $\mathrm{HO}_{1}$ mode propagation in these circunstances. The examination of Figs. (13) and (14) shows that the use of hyperbolic functions is profitable only for the centrally loaded configuration as in the side loaded configuration the conditions of single mode propagation and 
restriction to only real functions give the same answer. Theoretical charts for the centrally loaded configurations for samples requiring hyperbolic functions as shown in Figs. 15, 16, and 17 for the respective frequencies of 8,10 and $12 \mathrm{kmc} / \mathrm{s}$. Evaluation of loss tangent (tan $\delta$ )

The loss tangent is obtained from the measurements of $Q$ of the cavity system corresponding to two insertions of the sample differing by half a wavelength or any multiple of it inside the partially filled portion of the cavity. In fact, the process of evaluating the loss tangent from these measurements is broken up into two distinct steps, i.e., first the value of attenuation inside the partially filled portion is obtained which is later correlated to the loss tangent of the sample.

The reasons for selecting penetrations differing by half a wavelength can be understood by considering the sources of loss inside the cavity system. These sources of loss are outlined below:

(i) Losses occuring on the cavity's sidewalls $\left(=\mathrm{P}_{1}\right)$

(ii) Losses occuring on the cavity's end walls $\left(=\mathrm{P}_{2}\right)$

(iii) Losses occuring through the coupling holes $\left(=\mathrm{P}_{3}\right)$

(iv) Losses in the material under test $\left(=\mathrm{P}_{4}\right)$

(v) Losses due to the creation of higher order evanescent modes at the discontinuity $\left(=\mathrm{P}_{5}\right)$

From the general definition of $Q$ factor,

$$
Q=\omega \times \frac{\text { Total stored energy in the cavity }}{\text { Power lost in the cavity }}
$$

where $w$ is the angular frequency.

For the present cavity system

$$
Q=\omega \times \frac{W_{1}+W_{2}}{P_{1}+P_{2}+P_{3}+P_{4}+P_{5}} \cdots
$$

where $w_{1}$ is the power stored in the empty portion of the cavity while $w_{2}$ is the power stored in the partially filled portion of the cavity. 
$-13-$

Now by inserting the dielectric rod an integral number of half wavelengths, the cavity's resonance condition is not disturbed and it remains resonant at the same frequency. Since in the present condition, the fields at the junction between the two dielectric portions and the end walls remain the same and as the coupling holes are generally kept sufficiently away from the end of the dielectric specimen inside the cavity, all the three power losses designated by $P_{2}, P_{3}, P_{5}$ remain unchanged. The change in $Q$ results chiefly from the changes in $P_{1}$ and $\mathrm{P}_{4}$ which will be called $\mathrm{P}_{1}^{\prime}$ and $\mathrm{P}_{4}^{\prime}$ for this new configuration and from the changes in the stored energy $\left(w_{1}+w_{2}\right)$. which is now denominated as $\left(w_{1}^{\prime}+w_{2}^{\prime}\right)$. Hence $Q$ for this case, say $Q^{\prime}$, is given by

$$
Q_{1}^{\prime}=\omega \times \frac{W_{1}^{\prime}+W_{2}^{\prime}}{P_{1}^{\prime}+P_{4}^{\prime}+P_{2}+P_{3}+P_{5}}
$$

Hence,

$$
\left(P_{1}^{\prime}+P_{4}^{\prime}\right)-\left(P_{1}+P_{4}\right)=\omega\left\{\frac{W_{1}^{\prime}+W_{2}^{\prime}}{\varphi^{\prime}}-\frac{W_{1}+W_{2}}{Q}\right\} \ldots
$$

All these $P$ and $w$ terms have been expressed in terms of the various parameters of the cavity including the attenuation in the empty and partially filled portions and the following equations have been obtained correlating the attenuation with the measured $Q$ factors. It will be shown in the appendix that:

$$
\begin{aligned}
& \left.\frac{\omega_{r}}{2} \frac{\left[x+z_{2} n \lambda_{2 / 2} d \beta_{2 / d \omega}-z_{1} n \lambda_{1 / 2} d \beta_{1 / d \omega}\right.}{Q^{\prime}}-\frac{x}{\varphi}\right] \\
& =z_{2} \alpha_{2} n \lambda_{2 / 2}-z_{1} \alpha_{1} n \lambda_{1 / 2}-\ldots \ldots \text { (1) }
\end{aligned}
$$

where, $x=\left[z_{1} d_{1}^{\prime} \sec ^{2} \beta_{1} d_{1}^{\prime} d \beta_{1} / \alpha \omega+z_{2} l_{1} \sec ^{2}\left(\beta_{2} l_{1}^{\prime}\right) d \beta_{2} / d \omega\right.$

$$
\left.+\tan \left(\beta_{1} d_{1}^{\prime}\right) \cdot d z_{1 / d \omega}+\tan \left(\beta_{2} l_{1}^{\prime}\right) \cdot \frac{d z_{2}}{d \omega}\right]
$$


where $l_{1}^{\prime}=l_{1}+l_{0}$ and $d_{1}^{\prime}=d_{1}+d_{0}$, following the convention of Equation (3). The terms $z, \lambda, \alpha$ and $\beta$ stand respectively for wave impedance, guide wavelength, attenuation and phase constants. The suffix (1) attached to these terms refers to these quantities belonging to the empty portion of the cavity while the suffix (2) refers to the partially filled portion of the cavity. $d_{1}$ and $l_{1}$ refer to the lengths of the empty and the partially filled portions corresponding to the first measurement of $Q$ when the sample was not inserted by an integral number of half wavelengths. The equation (18) simplifies considerably if one starts with a completely empty cavity, i.e., $l_{1}=0$. In that case, the resonance condition simplifies to

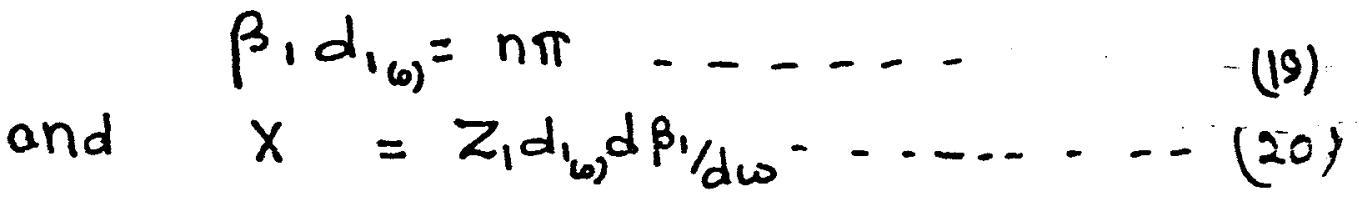

where $d_{1_{(0)}}$ is now the total length of the cavity.

It is possible to evaluate every term on the left hand side of Eq. (15) while $\alpha_{1}$ can be obtained from a separate experiment, hence $\alpha_{2}$ can be determined. It needs to be pointed out that the expressions (17) and $(18)$ are applicable to both the configurations under consideration. It has been shown in the appendix that $\alpha_{2}$ is related to $\tan \delta$ by the following expressions. 
$-15-$

The Side Filled Case

$\omega^{3} \mu_{0}^{2} \epsilon_{r} \epsilon_{0} K_{2}^{2} \tan \delta b t\left\{\phi_{0}\left(\phi_{0} t^{2} K_{1}^{2}-1\right)+1\right\}$

$$
\begin{aligned}
& +2 R_{m}\left[2 b k_{1}^{2} k_{2}^{2}+\beta^{2} \cdot \phi_{1}\left(\phi_{1}-1\right)+a \phi_{2} k_{1}^{2}\right. \\
& \begin{array}{l}
\alpha_{2}=\frac{k_{1}^{2} k_{2}^{2} t^{2} \phi_{0}^{2}\left\{\phi_{1} t+\phi_{2} a+\phi_{13} b\right\}}{2 \omega \mu_{0} \beta^{b}\left[\phi_{1} t\left(\phi_{0}-1\right)+a k_{1}^{2}\left(1+k_{2}^{2} \phi_{0}^{2} t^{2}\right)\right]} \\
\text { where } \quad \phi_{0}=\tan k_{1} t / k_{1} t \ldots . . .
\end{array} \\
& \phi_{1}=k_{1}^{2}-k_{2}^{2} \ldots \ldots \text { (23) } \\
& \phi_{2}=k_{2}{ }^{2}+\beta^{2} \cdots \cdots--(24) \\
& \phi_{3}=k_{1}{ }^{2}+k_{2}{ }^{2} \ldots \ldots \ldots \ldots
\end{aligned}
$$

$R_{m}$ is the resistivity of the material of the wall of the cavity and is related to $\alpha_{1}$ by the expression,

$$
R_{m}=\frac{\alpha_{1}}{T} \ldots \ldots \text { (26) }
$$

where $T$ is given by

$$
T=\sqrt{\epsilon_{0} / \mu_{0}} \times \frac{1}{b} \frac{1+2 b / a\left(\lambda_{0 / 2 a}\right)^{2}}{\sqrt{1-\left(\lambda_{0 / 2 a}\right)^{2}}} \ldots \text { (27) }
$$

where $\lambda_{0}$ is the free space wavelength and $b$ is the narrow dimension of the guide.

All the other terms in the equation (21) have the same meaning as used earlier in regard to the side filled case.

The computations needed to evaluate $\tan \delta$ from the measurements of $Q$ with the use of Eqs. (17) and (21), even though simpler as compared to the corresponding equations in the earlier paper are nonetheless fairly lengthy. However, the evaluation of $\tan \delta$ from $Q$ measurements is very considerably 
$-16-$

simplified by making the following approximations:

(i) The total energy stored in the cavity remains unaltered corresponding to the two insertions of the dielectric specimen;

(ii) The quantity $\left(\alpha_{2 \text { wall }}-\alpha_{1} \frac{z_{1}^{2}}{z_{2}^{2}}\right)$ is negligible compared to $\alpha_{2(\text { dielectric) }}$ (where $\alpha_{2 \text { (dielectric) }} \alpha_{2 \text { wall }}=\alpha_{2}$ )

By using the above approximations it will be shown that if one starts from zero insertion when $x=z_{1} d_{1} d \beta_{1} / d w$ and goes to an insertion of half a wavelength inside the cavity, $\tan \delta$ may be evaluated from:

$$
\left.\frac{1^{\prime}}{\phi^{\prime}}-\frac{1}{\Phi}=\frac{k_{2}^{2} \lambda_{2}^{2} \beta_{1} t_{r} t \tan \delta\left\{\phi_{0}\left(k_{1}^{2} t^{2} \phi_{0}-1\right)+1\right\}}{2 \beta_{2} \lambda_{1} d_{1(0)}\left\{\phi_{1} t\left(\phi_{0}-1\right)+a k_{1}^{2}\left(1+k_{2}^{2} \phi_{0}^{2} t^{2}\right)\right.}\right\}
$$

where $d(0)$ is the total length of the cavity resonance corresponding to zero insertion of the cavity.

Both the approximations mentioned above are justifiable. Approximation (1) is valid due to the fact that the total length of the cavity is very much longer than half a wavelength in the partially filled portion (it may be made equal to six wavelengths or more long without any mechanical problems due to the inherent simplicity of the arrangement) and hence the difference in the stored energy in the half wavelength long section, introduced due to the dielectric insertion is pretty negligible compared to the total stored energy of the cavity. It may easily be shown from the arguments used in the derivation of the expression for $\alpha_{2}$ that the ratio of this energy difference to the total, stored energy of the empty cavity is given by:

$$
\frac{\Delta W}{W}=\frac{1}{2} \frac{\left(\frac{z_{2}}{z_{1}} \lambda_{2} \frac{d \beta_{2}}{d \omega}-\lambda_{1} \frac{d \beta_{1}}{d w}\right)}{d_{1(0)} \frac{d \beta_{1}}{d \omega}}
$$


Equating the above expression to zero as stipulated in the approximation (1), for a typical set of values of $\lambda_{1}, \lambda_{2}, z_{1}, z_{2}, d \beta_{2} / d w$ and $\mathrm{d} \beta_{1} / \mathrm{dw}$ in a cavity which iA five wavelengths long, results in an error of less than $1 \%$ in the evaluation of $\alpha_{2}$. This error can be reduced in Linear Whe manner by further increasing the length of the cavity -- a requirement A

which can be met simply.

The second approximation introduces an error due to the neglecting of the quantity $\left(\alpha_{2(\text { wall })}-\alpha_{1} \frac{z_{1}^{2}}{z_{2}^{2}}\right)$ in comparison to $\alpha_{2 \text { (dielectric) }}$ As a first approximation one may assume the wall losses arising due to the imperfect conductivity remain unchanged even after the insertion of the dielectric, i.e., $\alpha_{2 \text { wall }}=\alpha_{1}$. In addition for the usual samples, the change in the value of the wave impedance of the filled section of the cavity after insertion of dielectric sample is of the order of 2 to $4 \%$, i.e., $z_{2} \simeq z_{1}$. Hence, the quantity $\alpha_{2 w a 11}-\left(\frac{z_{1}}{z_{2}}\right)^{2} \alpha_{1}$ in general turns out to be smaller than $\alpha_{1}$ by an order of magnitude or even more. Besides, for a well silvered surface, if the insertion of the sample into the cavity, by a length equal to a half wavelength, results in the lowering of $Q$ of the cavity by $1 / 3$ rd of its value, $\alpha_{2 d i e l e c t r i c}$ turns out to be an order of magnitude larger than $\alpha_{1}$. Hence, for such a case, the error introduced by the second approximation is less than $2 \%$. Hence, In general if one uses a cavity made from a long length of well silvered waveguide, the use of the approximate equation $(28)$ results in an error of less than five percent in the evaluation of $\tan \delta$. 
$-18-$

This sacrifice in accuracy, which is acceptable for most of the dielectric measurements, results however, in an enormous reduction of computational work and the elimination of the need to evaluate $\alpha_{1}$ by a separate experiment.

The Centrally Filled Case

Using the same symbols as used earlier in connection with this par-

$$
\begin{aligned}
& \text { ticular configuration } \\
& \omega^{3} k_{1}^{\prime 2} k_{2}^{\prime 2} \epsilon_{r} \epsilon_{0} \mu_{0}^{2} b t \tan \delta\left\{1+k_{1}^{\prime 2} d^{2} \phi_{0}^{\prime 2}-2 t^{\prime} d \phi_{0}^{\prime}\right\} \\
& +4 R_{m}\left\{k_{1}^{\prime 2}\left(1+k_{2}^{\prime 2} d^{2} \phi_{0}^{\prime 2}\right)\left(\phi_{2}^{\prime} d+k_{2}^{\prime 2} b\right)+k_{2}^{\prime 2} \phi_{3}^{\prime} t_{2}^{\prime}\left(1+d^{2} \phi_{0}^{\prime 2} k_{1}^{\prime 2}\right)\right. \\
& \left.\quad+\phi_{0}^{\prime} d\left(2 k_{1}^{2} k_{2}^{\prime 2}-\beta^{\prime 2} \phi_{1}^{\prime}\right)\right\} \\
& \alpha_{2}^{\prime}=\frac{\Delta \beta^{\prime} b \mu_{0} \omega\left\{k_{1}^{\prime 2} d+k_{2}^{\prime 2} \times t-\phi_{0}^{\prime} d \phi_{1}^{\prime}\right.}{\left.+k_{1}^{\prime 2} k_{2}^{\prime 2} \phi_{0}^{\prime 2} d\left(d+t_{2}\right)\right\}}
\end{aligned}
$$

where

$$
\begin{aligned}
& \phi_{0}^{\prime}=\frac{\tan k_{2}^{\prime} d}{k_{2}^{\prime} d} \ldots \ldots \ldots \ldots \\
& \phi_{1}^{\prime}=k_{1}^{\prime 2}+k_{2}^{\prime 2} \ldots \ldots \ldots \\
& \phi_{2}^{\prime}=k_{2}^{\prime 2}+\beta^{\prime 2} \ldots \ldots \\
& \phi_{3}^{\prime}=k_{1}^{\prime 2}+\beta^{\prime 2} \ldots \ldots \ldots
\end{aligned}
$$


once again, using the approximation mentioned earlier, one can obtain

$\frac{1}{\phi^{\prime}}-\frac{1}{\varphi}=\frac{k_{2}^{\prime 2} K_{1}^{\prime 2} t^{\prime} \lambda_{2}^{\prime 2} \beta_{1}^{\prime} \epsilon_{r} \tan \delta\left(1+k_{1}^{\prime 2} d^{2} \phi_{0}^{\prime 2}-2 / t^{d \phi_{0}^{\prime}}\right)}{4 d_{1(0)} \lambda_{1}^{\prime} \beta_{2}^{\prime}\left[k_{1}^{\prime 2} d+k_{2}^{\prime 2} \cdot t^{\prime}-\phi_{0} d \phi_{1}^{\prime}+k_{1}^{\prime 2} k_{2}^{\prime 2} d\left(d+t^{\prime} / 2\right)\right]-(35)}$

Experimental Resuits

Measurements of permittivity have been made on various samples of teflon, plexi-glass and a vew ceramics, with values of $\epsilon_{I}$ ranging up to 15. Some of these measurements have been made at the frequency of $10 \mathrm{Kmc}$ for which tables have been computed and the values of the parameters read directly off there. These values have been found to be in good agreement with the values available from other sources.

Typical curves of $(d+l)$ vs. $l$ obtained for teflon, both for the. side - loaded and the centrally loaded configurations have been shown in Figs. (18) and (19) respectively. The values of $\varepsilon_{x}$ obtained from both of these were in excellent agreement.

The $(d+l)$ vs $l$ curve obtained for a thick sample of plexi-glass $\left(t=.195^{\prime \prime}\right)$, in a centrally loaded configuration, for which the guide wavelength turns out to be smaller than the free space wavelength is shown in Fig. (20).

The $Q_{I}$ vs $\ell$ curve for a side loaded configuration (teflon, $t=.03^{\prime \prime}$ ) is shown in Fig. (21). The corresponding curve for the centrally loaded configuration was also similar.

The value of $\tan \delta$ was obtained by taking a number of different pairs of points from $Q_{L}$ vs 2 curve and the agreement in $\tan \delta$ value has been of the order of the estimated accuracy of $Q$ measurements.

In general, the accuracy of measurement for permittivity is within $1 \%$ and the accuracy of measurement of $\tan \delta$ is within $\pm 2 \%$. However, if the approximate expression for the evaluation of $\tan \delta$ be used, the estimated accuracy of measurement of $\tan \delta$ is within $\pm 7,3$. 


\section{$-20-$}

Discussion of Results and Conclusions

(1) From the results obtained it follows that this method is capable of giving accurate and reliable results for the required parameters and the accuracy obtained by this is comparable or better than the accuracy obtained by the other methods.

(2) It may once again be stated that all the major advantages reported in the earlier paper are retained here as enumerated below.

(i) The method of permittivity measurement has to rely on the use of a large number of datum points and as such this is a precision method in the (15) sense defined by olinerand Altschuler. Also, tan $\delta$ may be determined from many pairs of results.

(ii) From the very nature of the arrangements used, there is a wide choice in the values of the thicknesses of the samples that could be investigated. This technique because of the possibility of using two different and the possibility of using hyperbolic functions for centrally loaded configuration configurations gives a wide range in the values of the permittivities that could be measured.

(iii) All the measurements are made at a fixed frequency which considerably simplifies the experimental work.

(iv) Once again, the errors introduced due to the uneven finish of the (ID) end of the sample in the cavity and the tilted end walls of the cavity are obviated.

(3) The relative simplicity in the computational efforts is a distinct advantage of this method over the previous one. For most of the requirements where the accuracy of $\pm 7 \%$ is adequate for tan $\delta$ measurement, the 
use of the approximate expression drastically reduces the computations for the evaluation of $\tan \delta$. In the majority of the cases, $\tan \delta$ will be given within $5 \%$ by the use of the approximate expression.

(4) The method does not suffer from the problems encountered with the excitation of unwanted modes into the cavity.

(5) It may be mentioned that this method may very profitably be used for investigating the magnetic dielectricglike ferrites by suitably combining the measurements on a thin ferrite sample, first by placing it along the sidewalls and then by placing it along the center. This technique with the facility to position the sample in the maxima of electric or magnetic field may be a very useful method for the investigation of thin films. Acknowledgements

Thanks are due to Dr. N. B. Bhatt, Director, Solid State Physics Lab., Delhi, for permission to start this work, and also to Mr. G. P. Sharma for his help in the initial stages of this work. Thanks are particularly due to Professor H. C. Bourne, Jr. Chairman, Electrical Engineering Department, Rice University for making available the facilities of the laboratory and workshop of his department where most of the reported work has been done. 


\section{References}

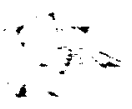

(1) Lamb, J., "Dielectric Measurement at Wavelength Around $1 \mathrm{~cm}$ by Means of an $\mathrm{HO}_{1}$ Cylindrical Cavity Resonator", Proceedings IEE, Vol. 93, 1946, Pt. III A, P. 1447.

(2) Horner, F , Taylor, T. A. Dunsmiir, R., Lamb, J., and Jackson, W.,

"Resonance Methods of Dielectric Measurements at Centimetric Wavelengths", Ibid 1946, 93, Pt. III, p. 53.

(3) Collie, C. H., Hasted, J. B. and Ritson, D: M., "The Cavity Resonator Method of Measuring the Dielectric Constants of Polar Liquids in the Centimeter Band", Proceedings Physical Society, Vol. 60, 1948, p. 71.

(4) Sinha, J. K., and Brown, J., "A New Cavity Resonator Method of Measuring Permittivity", Proc. IEE, Vol. 107, Part B, Nov. 1960, p. 522.

(5) Hotston, E.S., "Correction Terms for Dielectric Measurements with Cavity Resonators", J. of Scientific Instruments, Vol. 38, 1961, p. 13

(6) Feenberg, E., "Relation Between Nodal Positions and Standing Wave Ratio in a Complex Transmission Systems, J. of Applied Physics, Vol. 17, No. 6, 1946, p. 530 .

(7) Marcuvitz, N., "Waveguide Handbook", Radiation Laboratory Series, Vol. 10, McGraw Hil1, 1951, Chapter 3.

(8) Collin, R. E. \& Brown, J., "The Calculation of the Equivalent Circuit - of an Axially Unsymetrical Waveguide Junction", Proceedings IEE, Monograph No. $145 \mathrm{R}$, August 1955 (103C, p. 121).

(9) Sinha, J.K., "A Method for the Evaluation of Equivalent Circuit Parameters of an Asymetric Waveguide Junction", Ijid, Monograph No. 381E, May 1960.

(10) Pincherle, L. "Electromagnetic Waves in Metal Tubes Filled Longitudinally with Two Dielectrics, Physical Review, 1944, 66, p. 118. 
(11) Collins, R. E., "Field Theory of Guided Waves", McGraw-Hill Book Co. 1960 , P. 228

(12) Vartanian, P. H., Apres, E.P. \& Gelgesson, A. L., "Propagation in Dielectric Slab Loaded Rectangular Wave Guide", IRE Trans. on Microwave Theory \& Techniques, April 1958, p. 215.

(13) Montgomery, C. G., Dicke, R. H. \& Purcell, E.M.,"Principles of Microwave Circuits", Chpt. 2, Radiation Laboratory Series, Vol. 8, 1948.

(14) Sinha, J.K., "A New Cavity Resonator Method of Measuring Dielectric Constants", London University, Ph.D. Thesis, 1959.

(15) Oliner, A.A. = Altschuler, H.M., "Methods of Measuring Dielectric Constants Based on Microwave Network viewpoint", Journal Applied Physics, Vol. 26, 1955, p. 214

\section{Appendices}

1. Evaluation of the Total Stored Energy $\left(W_{1}+W_{2}\right)$ and the Losses $\left(P_{1}+P_{2}\right)$ The steps required in deriving the Eq. (17) from Eq. (16) for expressing the attenuation $\alpha_{2}$ in terms of $Q$ and $Q^{\prime}$ are given below.

For the evaluation of stored energy $\left(w_{1}+w_{2}\right)$, the cavity system is represented by the equivalent circuit shown in Fig. (22). The lengths $d_{1}$ and $l_{i}$ as shown are different from the corresponding physical lengths by the amounts $d_{0}$ and $\ell_{0}$, respectively, due to the phase effects introduced at the junction. As the cavity system is being considered at resonance, the equivalent transmission system is short circuited at both the ends.

For calculating the stored energy, the two sections shown in the circuit of Fig. 2, having different propagation constants are considered separately. Consider now the section to the right of the junction plane A shown in Fig.(23) and 
$-24-$

choose the coordinates in such a manner that the distances away from the short circuit are considered negative while the short circuit is at the plane $\mathrm{z}=0$. $\mathbf{S}_{2}$ represents the cross sectional area of the system at the plane $A$ and $n$ is the direction normal to $S_{2}$.

For the system under consideration if $\mathrm{E}_{2}$ and $\mathrm{H}_{2}$ represent the transverse components of the electric and magnetic field at the plane $A$, it can be shown that:

$$
E_{2}(\text { trans })=D_{2} \sin \left(\beta_{2} z\right) \underline{e}_{2}(x, y)
$$

and

$$
\mathrm{H}_{2} \text { (trans) }=\mathrm{jY}_{2} \mathrm{D}_{2} \cos \left(\beta_{2} \mathrm{Z}\right) \underline{\mathrm{e}}_{2}(\mathrm{x}, \mathrm{y})
$$

In the above equations $D_{2}$ and $Y_{2}$ are respectively the amplitude coefficient and the wave impedance while $e_{2}(x, y)$ is the function giving the variation of the fields in the $x, y$ plane.

It can be shown ${ }^{13}$ by proper operations on the Maxwell equation that:

$$
\int(E X \delta H-\delta E X H) \cdot \underline{n} d s=-j 4 \delta \omega_{\gamma} \times \text { Total stored energy }
$$
(38)

Applying this result to the system under consideration, it is seen that the only contribution to the surface integral $s$ as shown in the Eq. (38), arises due to the cross section area $S_{2}$ at the plane A. After carrying through the mathematical operations, it is found that the energy $\mathrm{W}_{2}$ stored In the section to the right of plane $A$ as shown in Fig. (16) is given by

$$
W_{2}=\frac{1}{4} D_{2}^{2}\left\{l_{1}^{1} Y_{2} d \beta_{2} / d \omega-\frac{d Y_{2}}{d \omega} \frac{\sin 2 \beta_{2} l_{1}^{\prime}}{2} \int_{s_{2}} e_{2}^{2} d s_{2} \cdot-(39)\right.
$$


$-25-$

Similarly, the energy stored in the section shown to the left of plane A in Fig. (15) is given by

$$
W_{1}=\frac{1}{4} D_{1}^{2}\left\{d_{1}^{\prime} Y_{1} d \beta_{1} / d \omega-\frac{d \psi_{1}}{d \omega} \frac{\sin 2 \beta_{1} d_{1}^{\prime}}{2}\right\} \int_{s_{1}} e_{1}^{2} d s_{1} \ldots .(40)
$$

Before writing the expression for the total energy $\left(w_{1}+w_{2}\right)$, it is convenient first to find the relation between the amplitude constants $D_{1}$ and $D_{2}$ in the two sections by applying the Pointing Theorem which is expressed for a lossless cavity as:

$$
\int_{s}(\underline{E X H}) \cdot n \cdot d s=\frac{1}{2} j \omega \int_{v}\left(\mu|H|^{2}-\epsilon \mid \underline{|E|^{2}}\right) d v
$$

where $S$ is the surface area and $V$ the volume of the cavity, but $S$ being a chased conductor in the case of a cavity. Eq. (4I) is equal to zero, hence the total stored magnetic energy is equal to the total stored electric energy.

Applying these results to the system under consideration, it is found that

$$
\int_{S_{1}}\left(\underline{E}_{1} \times \underline{H}_{1}\right) \cdot n d s_{1}=-\int_{s_{2}}\left(\underline{E_{2}} \times \underline{H}_{2}\right) \cdot n d s_{2}
$$

Putting in the values of $\mathrm{E}_{2}, \mathrm{H}_{2}$ from equations $(36)$ and $(37)$, the eq. $(42)$ gives

$$
\frac{D_{2}^{2}}{D_{1}^{2}} \frac{\int_{S_{2}} e_{2}^{2} d s_{2}}{\int_{S_{1}} e_{1}^{2} d s_{1}}=-\frac{\sin \left(2 \beta_{1} d_{1}^{\prime}\right)}{\sin \left(2 \beta_{2} l_{1}^{\prime}\right)} \cdot \frac{Y_{1}}{Y_{2}} \cdots \cdot
$$


$-26-$

Using these results together with the result of Eq. (3), the total energy $W=W_{1}+W_{2}$ is given by:

$$
\begin{aligned}
\cdot W_{1}+W_{2} & =\frac{1}{8} D_{1}^{2} Y_{1}^{2} \frac{\sin \left(2 \beta_{1} d_{1}\right)}{\tan \left(\beta_{1} d_{1}\right)}\left[z_{1} \operatorname{Sec}^{2}\left(\beta_{1} d_{1}\right) d_{1}^{\prime} \frac{d \beta_{1}}{d \omega}+z_{2} l_{1}^{\prime} \sec ^{2}\left(\beta_{2} l_{1}^{\prime}\right) \frac{d \beta_{2}}{d \omega}\right. \\
& \left.+\tan \left(\beta_{1} d_{1}^{\prime}\right) d z_{1 / d \omega}+\tan \left(\beta_{2} l_{1}^{\prime}\right) \frac{d z_{2}}{d \omega}\right] \int_{S_{1}} e_{1}^{2} d s_{1}
\end{aligned}
$$

Consider now the case when the dielectric rod is pushed further inside by an amount equal to $\frac{\lambda_{2}}{2}$ and then this reduces by $\frac{\lambda_{1}}{2}$, in order to maintain resonance, for such a situation, as the fields remain undistorted, the amplerude constants $D_{1} \& D_{2}$ remain unchanged. If the new lengths be called $l_{1}^{\prime}$ and $d_{i}$, it can easily be seen that:

$$
\sec ^{2}\left(\beta_{1} d_{1}^{\prime}\right)=\sec ^{2}\left(\beta_{1} d_{1} \& \sec ^{2}\left(\beta_{2} l_{1}^{\prime}\right)=\sec ^{2} \beta_{2} l_{1}\right)
$$

Hence, carrying through the same arguments as before, it can be seen that the total energy $W_{1}^{\prime}+W_{2}^{\prime}$ in this configuration is given by

$$
\begin{aligned}
& W_{1}^{\prime}+W_{2}^{\prime}=\frac{1}{8} D_{1}^{2} Y_{1}^{2} \frac{\sin \left(2 \beta_{1} d_{1}^{\prime}\right)}{\tan \left(\beta_{1} d_{1}^{\prime}\right)} \int_{s_{1}^{\prime}} e_{1}^{2} d s_{1} \cdot\left[x+\frac{z_{2}}{2} \lambda_{2} \sec ^{2} \beta_{2} l_{1}^{\prime} d \beta_{2}\right. \\
& \left.-\frac{z_{1}}{2} \lambda_{1} \sec ^{2}\left(\beta_{1} d_{1}^{\prime}\right) \frac{d \beta_{1}}{d \omega}\right] \\
& \begin{aligned}
X= & \left.z_{1} d_{1}^{\prime} \sec ^{2}\left(\beta_{1} d_{1}^{\prime}\right) \frac{d \beta_{1}}{d \omega}+z_{2} l_{1}^{\prime} \sec ^{2} \beta_{2} l_{1}^{\prime}\right) \frac{d \beta_{2}}{d \omega} \\
& +t_{0 n}\left(\beta_{1} d_{1}^{\prime}\right) \frac{d z_{1}}{d \omega}\left(\beta_{2} l_{1}^{\prime}\right) d z_{2}
\end{aligned}
\end{aligned}
$$


$-27-$

Substituting the value of $W_{1}+W_{2}$ and $W_{1}^{\prime}+W_{2}^{\prime}$ from equations (44), (45) in Eq. (16) gives

$$
\begin{gathered}
\frac{1}{8} D_{1}^{2} Y_{1}^{2} \frac{\sin \left(2 \beta_{1} d_{1}^{\prime}\right)}{\tan \left(\beta_{1} d_{1}^{\prime}\right)} \int_{S_{1}} e_{1}^{2} d s_{1} x\left[\frac{x+\frac{z_{2} \lambda_{2}}{2} \sec ^{2}\left(\beta_{2} l_{1}^{\prime}\right) \frac{d \beta_{2}}{d \omega}-z_{1} \lambda_{1} \sec ^{2}\left(\rho d_{1}^{\prime}\right)}{\Phi_{2}}\right. \\
\left.\quad-\frac{x}{\Phi_{1}}\right] \\
\left.=\frac{1}{\omega} \text { (Transmission losses in length } \frac{\lambda_{2}}{2}-\text { transmission loss in } \frac{\lambda_{1}}{2}\right)(4 \mathrm{D}
\end{gathered}
$$

Consider now the losses in $\frac{\lambda_{2}}{2}$ section. This $\frac{\lambda_{2}}{2}$ section may be assumed to be equivalent to a cavity with zero end wall impedances, as only the transmission losses are to be evaluated. As the $Q$ of this section is quite high, it will be a perfectly valid assumption to take the impedance of this cavity to be zero at resonance. This gives

$$
\tanh \left(\gamma_{2} \lambda_{2 / 2}\right)=0
$$

$(48$

(49)

The $Q$ of this section may be evaluated by making the resonant firequince $\omega_{r}$ complex and equate it to $\omega_{r}^{\prime}+j p, \omega_{r}^{\prime}$ and $p$ being $i$ ts real and imaginary components respectively. It has been shown ${ }^{13}$ that $Q$ of the cavity is equal to

$$
Q=\frac{w_{r}}{2 p}
$$

Since $\omega_{r}$ is assumed to be complex, hence $\beta_{2}$ also becomes complex and equal to:

$$
\beta_{2}=\beta_{2}^{\prime}+j p \frac{d \beta_{2}}{d \omega}
$$

Hence

$$
\gamma_{2}=j \beta_{2}^{\prime}+\left(\alpha_{2}-p \cdot \frac{d \beta_{2}}{d \omega}\right)
$$


Substituting this value of $Y_{2}$ into Eq. (48) and using Taylor's expansion, one obtains

$$
p=\frac{\alpha_{2}}{d \beta_{2 / \alpha}}
$$

and therefore :

$$
q=\omega_{r} \frac{d \beta_{2} / d \omega}{2 \alpha_{2}}
$$

Using the definition of $Q$ as given in Eq. (13), it follows that the energy lost in the section $\frac{\lambda_{2}}{2}=2 \alpha_{2} x$

$$
\text { Energy stored in length } \lambda_{2 / 2} / d \beta_{2 / d \omega^{-}-}
$$

Using the expression for stored energy as given in Eq. (39), the transmission losses in $\frac{\lambda_{2}}{2}$ section $=\frac{1}{4} D_{2}^{2} \alpha_{2} \lambda_{2} Y_{2} \int_{S_{2}} e_{2}^{2} d s_{2}$ Similarly the transmission losses in

$$
\frac{\lambda_{1}}{2} \text { section }=1 / 4 D_{1}^{2} \alpha_{1} \lambda_{1} Y_{1} \int_{S_{1}} e^{2} d s_{1}-\cdots
$$

Hence, transmission Losses in length $\frac{\lambda_{2}}{2}$ - Transmission Losses in $\frac{\lambda_{1}}{2}=$

$$
\left.\frac{1}{4} D_{1}^{2} Y_{1}^{2} \int_{S_{1}} e_{1}^{2} d s_{1} \frac{\sin 2 \beta_{1} d_{1}^{\prime}}{\tan \beta_{1} d_{1}^{\prime}}\left[z_{2} \alpha_{2} \lambda_{2} \sec ^{2} \beta_{2} l_{1}^{\prime}\right)-z_{1} \alpha_{1} \lambda_{1} \sec ^{2} \beta_{1} d_{1}^{\prime},\right]
$$

Using the result of Eq. (58) in Eq. (47; , one obtains,

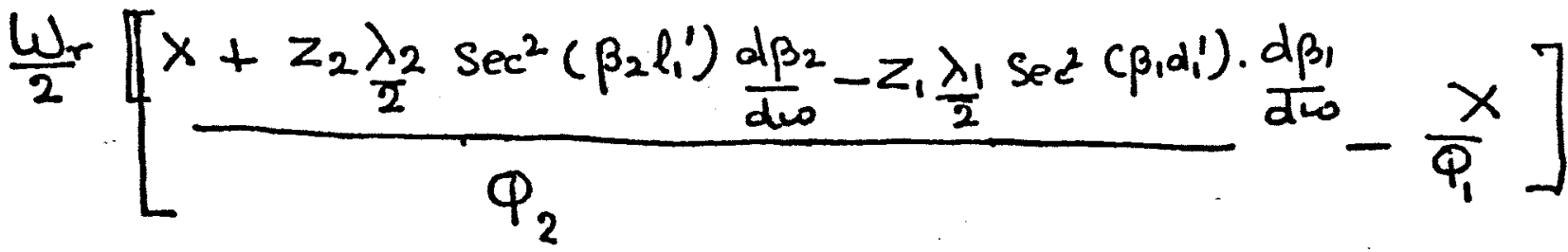

$$
\begin{aligned}
& \left.=Z_{2} \alpha_{2} \frac{\lambda_{2}}{2} \operatorname{Sec}^{2} \beta_{2} l_{1}^{\prime}\right)-Z_{1} \alpha_{1} \frac{\lambda_{1}}{2} \sec ^{2}\left(\beta_{1} d_{1}^{\prime}\right.
\end{aligned}
$$

It may be mentioned that the above analysis is also applicable if $\ell_{1}$ is changed by any multiple of $\lambda_{2}$ or by $n \lambda_{2}$. This results in the general form of the above equation as given in Eq. (18). 
$-29-$

As the equations (18) and (20) contain both the angles $\beta_{1} d_{1}$ and $\beta_{2} l_{1}^{\prime}$, for the simplicity in computations it is found desirable to eliminate one of the angles by using the Equation (3).

From the generality of the foregoing derivations it is easy to see that the above equations are valid both for the sidefilled and the end filled cases.

2. Evaluation of the terms $d \beta_{1} / d \omega$ and $d \beta_{2} / d \bar{\omega}$.

The term $\frac{d \beta_{1}}{d \omega}$ appearing in the above equation is evaluated very simply by differentiating the simple waveguide equation written below

$$
\beta_{1}^{2}=\omega^{2} \mu_{0} \varepsilon_{0}-K_{c}^{2}
$$

where $k_{\dot{c}}$ is a constant being equal to $\frac{2 \pi}{\lambda_{c}}$.

Hence

$$
\frac{d \beta_{1}}{d \omega}=\omega \mu_{0} \epsilon_{0} / \beta_{1}
$$

$\frac{d \beta_{2}}{d \omega}$ on the other hand is a more complicated function of $a, t, k_{1}$ and $\mathrm{K}_{2}$. Consider first the side filled case. Differentiating the equations (7) and (8:), one obtains:

$$
\beta_{2} \frac{d \beta_{2}}{d \omega}=\omega \mu_{0} \epsilon_{0}-k_{2} \frac{d k_{2}}{d \omega}=\omega \mu_{0} \epsilon_{1}-k_{1} \frac{d k_{1}}{d \omega}
$$

Similarly, differentiating Eq. ( 6$)$,

$$
\frac{d \phi_{1}}{d k_{1}}=-\frac{d \phi_{2}}{d k_{2}} \cdot \frac{d k_{2}}{d \omega}
$$

from the equations $\left(\sigma_{2}\right)$ and $\left(\sigma_{3}\right)$, it is easy to see that

$\omega \mu_{0} \varepsilon_{1}-\omega \mu_{0} \epsilon_{0}=\left[\frac{k_{1} \frac{d \phi_{2}}{d k_{2}}+k_{2} d \phi_{1}}{d \phi_{2} / d k_{2}} d k_{1}\right] \cdot \frac{d k_{1}}{d \omega}$ 
Putting the value of $\left(\frac{\mathrm{dK}_{1}}{\mathrm{~d} \omega}\right)$ from Eq. (54) into Eq. (62), one gets

$$
\begin{aligned}
& \frac{d \beta_{2}}{d \omega}=\frac{\omega \mu_{0} \epsilon_{0}}{\beta_{2}}\left[\frac{k_{1} \frac{d \phi_{2}}{d k_{2}}+k_{2} \epsilon_{r} \frac{d \phi_{1}}{d k_{1}}}{k_{1} \frac{d \phi_{2}}{d k_{2}}+k_{2} d \phi_{1 / d k_{1}}}\right] \ldots-(65) \\
& \text { where } \\
& \text { and } \quad \frac{d \phi_{1}}{d k_{1}}=\frac{t\left(k_{1} t\right) \sec ^{2}\left(k_{1} t\right)-t \cdot \tan \left(k_{1} t\right)}{k_{1}^{2} t^{2}} \ldots-(66) \\
& d \phi_{2 / d k_{2}}=\frac{(a-t) k_{2} t \sec ^{2} k_{2}(a-t)-t \tan k_{2}(a-t)}{k_{2}^{2} t^{2}}
\end{aligned}
$$
the Equations (9), (10) and (.It) and one obtains

where

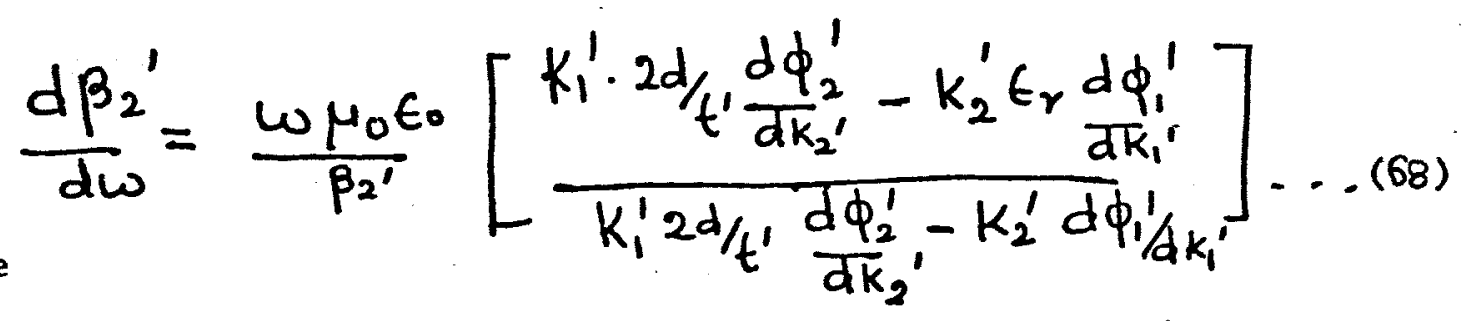

$$
\begin{aligned}
& \frac{d \phi_{1}^{\prime}}{d k_{1}^{\prime}}=-\left[\frac{k_{1}^{\prime} t^{\prime} \operatorname{cosec}^{2} k_{1}^{\prime} t_{2}^{\prime}+2 \cot k_{1}^{\prime} t^{\prime} / 2}{k_{1}^{\prime 2} t^{\prime}}\right]-{ }^{699)} \\
& \frac{d \phi_{2}^{\prime}}{d k_{2}^{\prime}}=\because\left[\frac{k_{2}^{\prime} d \sec ^{2}\left(k_{2}^{\prime} d\right)-\tan k_{2}^{\prime} d}{k_{2}^{\prime 2} d}\right] \ldots(70)
\end{aligned}
$$

3. Correlation of $\alpha_{2}$ with $\tan \delta$.

The attentuation constant $(\alpha)$ in a transmission system is defined

as

$$
\begin{aligned}
\alpha & =\frac{\text { Rate of power dissipation }}{2 \times \text { Power flowing in the }} \\
& =\frac{\mathrm{dP}}{\frac{\mathrm{dz}}{2 \mathrm{P}}} \quad . \quad
\end{aligned}
$$




\section{$-31-$}

For the case under consideration $\frac{d p}{d z}$ consists of two parts, $\frac{d P}{d z}$ (conductor) i.e., the power loss due to the finite conducting of the metallic walls and $\frac{d p}{d z}(d i e l)$, i.e., the power losses due to the dielectric sample itself. It is known that:

$$
\frac{d p}{d z \text { (conductor) }}=\frac{R_{m}}{2} \int_{s}|H t|^{2} d s \ldots
$$

where $R_{m}$ is the surface resistivity and $|H t|$ is the magnetic field which is tangential to the walls. The integration is carried out for the entire surface area/unit length of the transmitting guide.

$$
\frac{d P}{d z \text { (dielectis) }}=\frac{1}{2} \omega E_{r} \epsilon_{0} \tan \int_{v}|E|^{2} d v \ldots \ldots,
$$

$\tan \delta$ in the above equation is the loss tangent term to be evaluated from the value of $\alpha_{2}$ and the integration is done at the volume occupied by the dielectric specimen per unit length.

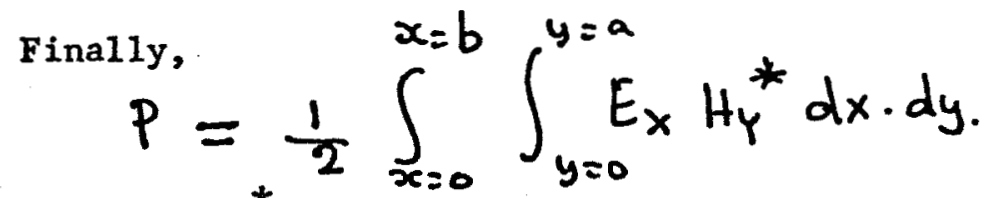

where $E_{x}$ and $\mathrm{Hy}^{*}$ are the tangential components of the fields and the integration is carried out over the cross section of the waveguide.

Putting the values of the fields :

the Equations (73), (74) and (75) one obtains the correlation of $\alpha_{2}$ with $\tan \delta$ for the sidefilled case as given in Equation (2) $)$.

Similarly, putting the values of the fields:

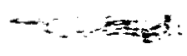
$\therefore \quad$ in the Equations $(73),(74)$ and $(75)$, one obtains the corresponding expression for $\alpha_{2}$ in terms of $\tan \delta$ for the centrally filled case as given in equation (28). 
4. Derivation of the approximate relation for the evaluation of $\tan \delta$.

If the first measurement of $Q$, say $Q_{1}$, corresponds to zero insertions and the second measurement, say $Q_{2}$, to half wavelength insertion, it follows then from Eq. (17), ignoring the difference in stored energy in the two situations:

$$
\frac{\omega_{r}}{2}\left(\frac{X}{Q_{2}}-X / \Phi_{1}\right)=Z_{2} \alpha_{2} \frac{\lambda_{2}}{2}-z_{1} \alpha_{1} \frac{\lambda_{1}}{2}
$$

Now putting the value of $x=\frac{z_{1} d_{1(0)}{ }^{\omega \mu_{0}} \epsilon_{0}}{\beta_{1}}$ from equations (20) and (BI), when $d_{1(0)}$ is the resonant length of the empty cavity

$$
d_{1(0)} \frac{\omega^{2} \mu_{0} \varepsilon_{0}}{\beta_{1}}\left(\frac{1}{Q_{2}}-\frac{1}{Q_{1}}\right)=\frac{Z_{2}}{\bar{z}_{1}} \alpha_{2} \lambda_{2}-\alpha_{1} \lambda_{1}
$$

Hence

$$
\frac{Z_{1}}{Z_{2}} \frac{\omega^{2} \mu_{0} \varepsilon_{0}}{\lambda_{2}} d_{(0)}\left(\frac{1}{\Phi_{2}}-\frac{1}{\Phi_{1}}\right)=\alpha_{2}-\alpha_{1} \frac{z_{1}}{z_{2}} \frac{\lambda_{1}}{\lambda_{2}} \ldots \text { (78) }
$$

Now if $\alpha_{2}$ is written as $\alpha_{2(\text { wall) }}+\alpha_{2(\text { diel), }}$, one easily obtains the Eq. (28) from the Eqs. $(Q V)$ and $(7,5)$, where $Q^{\prime}$ and $Q$ correspond to $Q_{2}$ and $Q_{1}$, respectively, if $\left\{\alpha_{2(\text { wall })}-\alpha_{1} \lambda_{1}{ }^{2} / \lambda_{2}{ }^{2}\right\}$ be ignored in comparison $\alpha_{2}$ (dielectric).

5. As mentioned earlier, $Q$ of the cavity is obtained at a fixed frequency by the tuning plunger by keeping $\ell$ fixed and varied. The relation between $\delta d$ and $\delta f$, the corresponding frequency charge, is obtained by differentiating Eq. (3) which gives

$$
\begin{aligned}
& \frac{\delta f}{\delta d}= \frac{\beta_{1}\left[\frac{z_{2}^{2}}{z_{1}^{2}} \sec ^{2}\left(\beta_{2} l^{\prime}\right)+\left(\frac{z_{1}^{2}-z_{2}^{2}}{z_{1}^{2}}\right)\right]}{\sec ^{2}\left(\beta_{2} l^{\prime}\right)\left\{\frac{z_{2}}{z_{1}} \cdot l^{\prime} d \beta_{2}\right.} \frac{z_{2}^{2}}{d f} d^{\prime} d \beta_{1} / d f \\
& z_{1}^{2} \\
&+\tan \left(\beta_{2} l^{\prime}\right) \frac{d}{d f}\left(z_{2} z_{1}\right)+\left(\frac{z_{1}^{2}-z_{2}^{2}}{z_{1}^{2}}\right) \cdot \frac{d \beta_{1}}{d f}
\end{aligned}
$$




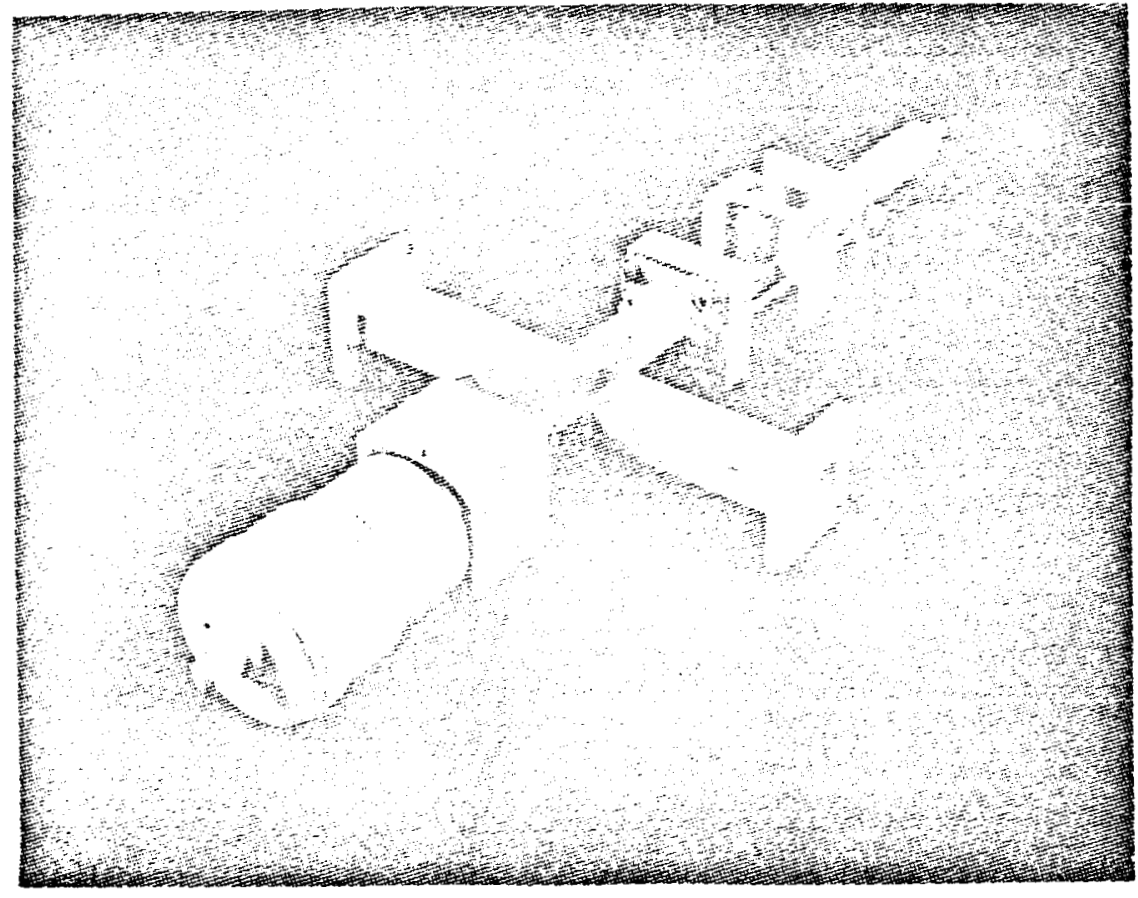

Fig. I - Photograph of the Cavity System 


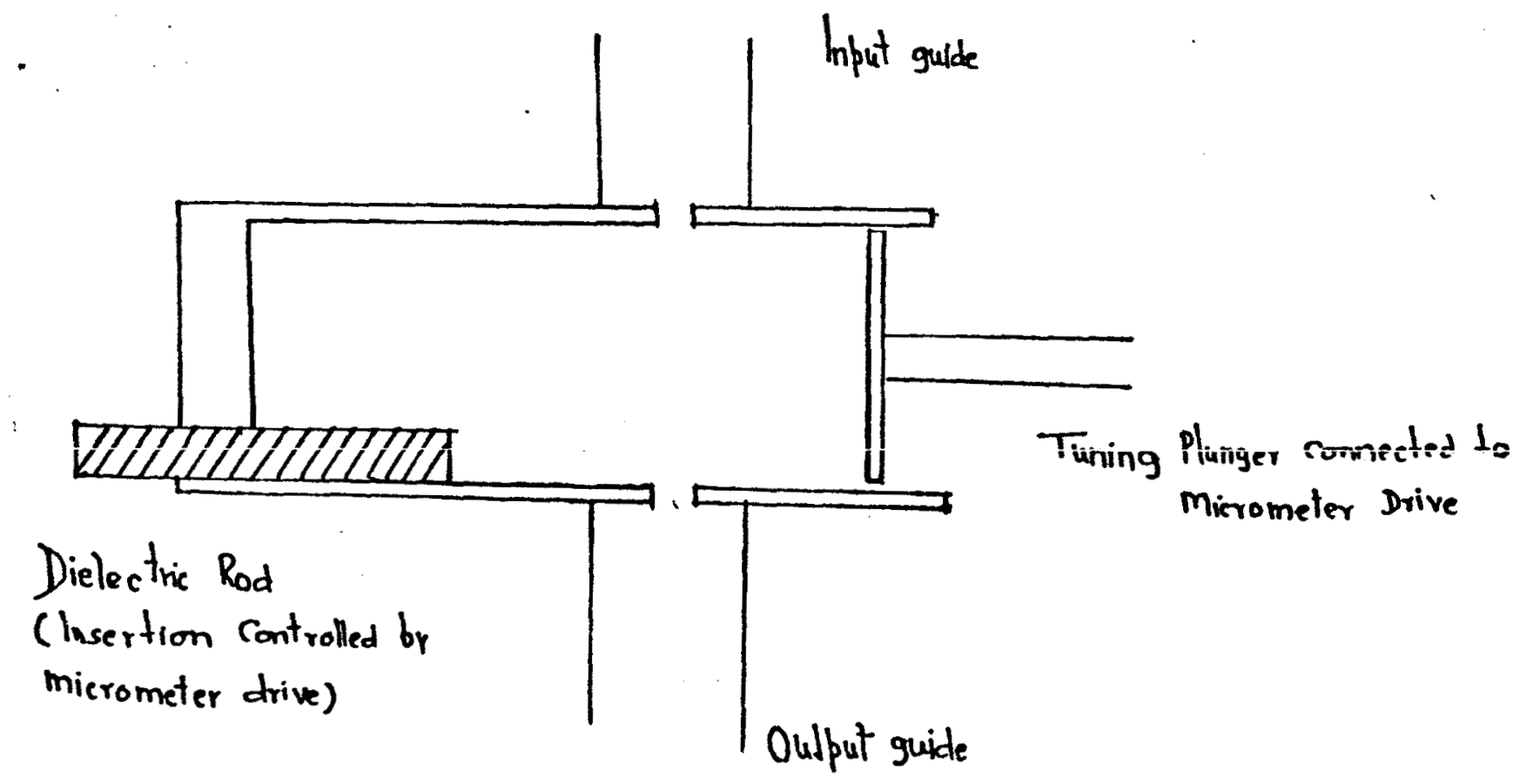

Fig 2. - General arrangement of the cavity 8 the sample

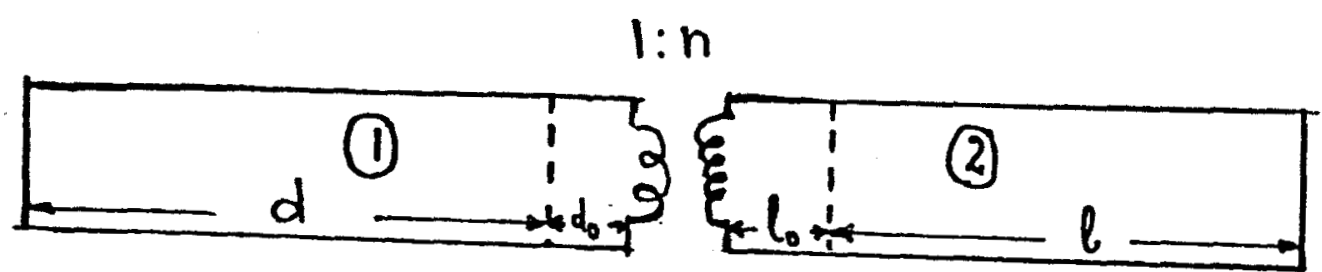

Fig - Equivalent circuit for the cavity

(Transmission lines (1) \& (2) represent the Hor modes in the emply $\&$ partially filled portions of the cavity respectively) 


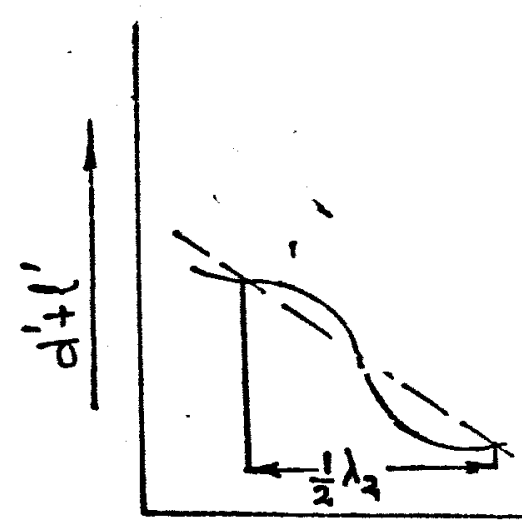

Fig 4 -General shape of the curve showing the dependence of $\left(d^{\prime}+l^{\prime}\right)$ on $l^{\prime}$.
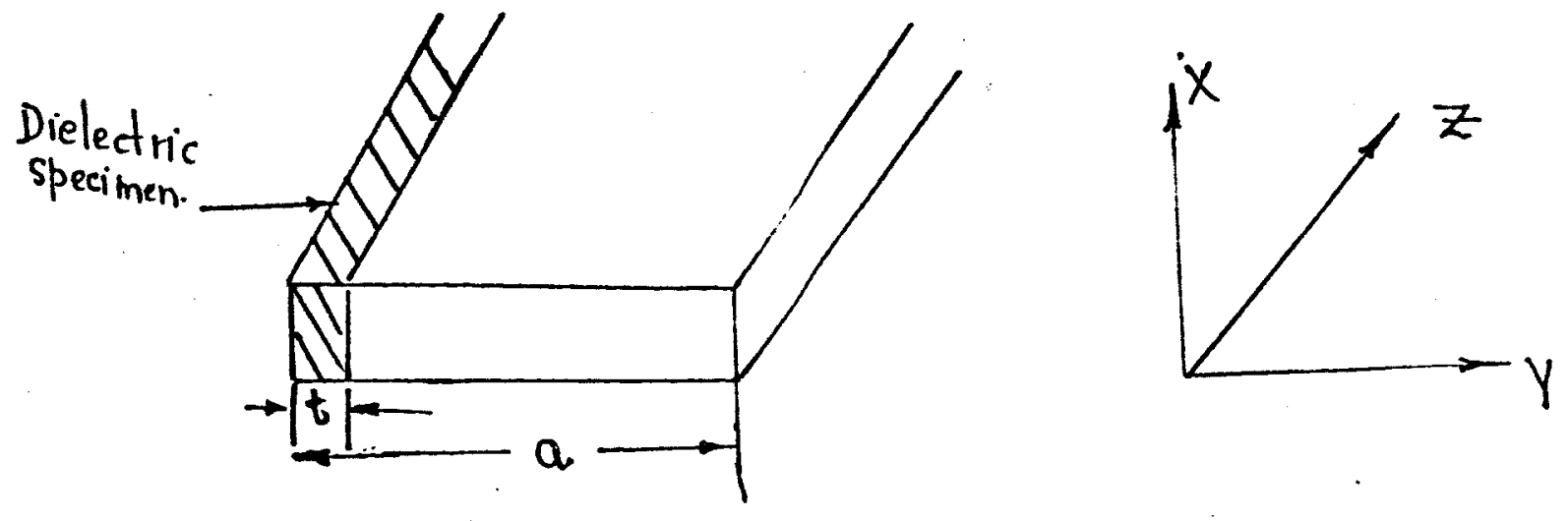

Fig 5 - Arrangement showing the sample along the cavity's side wall. 


$$
\begin{aligned}
& \text { 足 }
\end{aligned}
$$

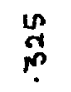

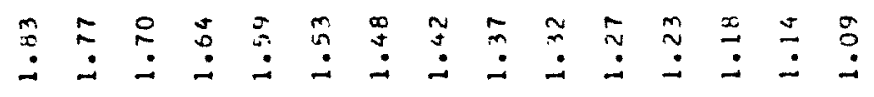

$$
\begin{aligned}
& \stackrel{\circ}{\circ}
\end{aligned}
$$

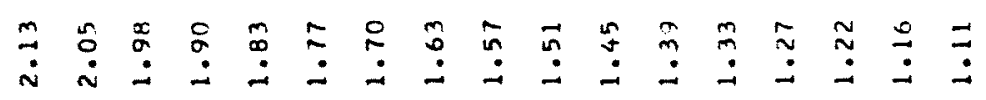

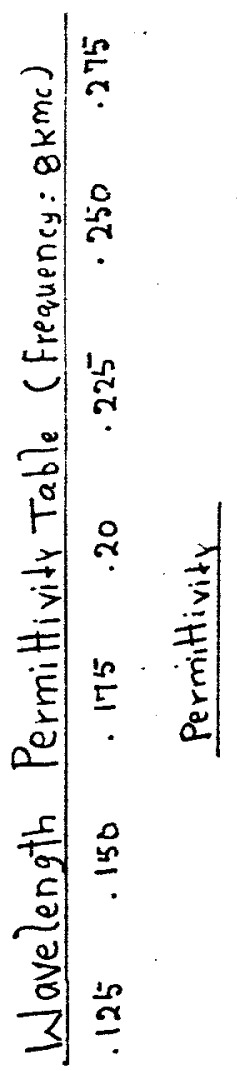

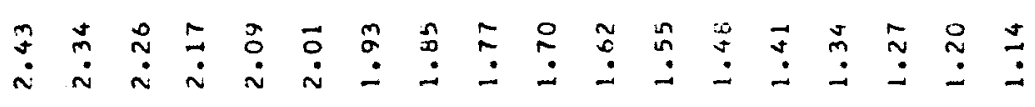

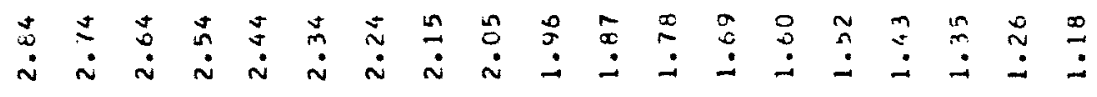

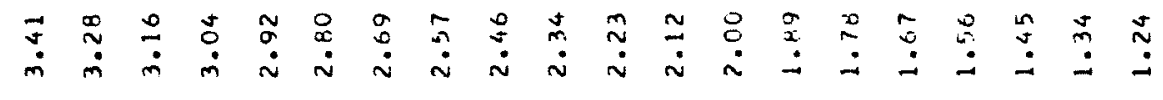

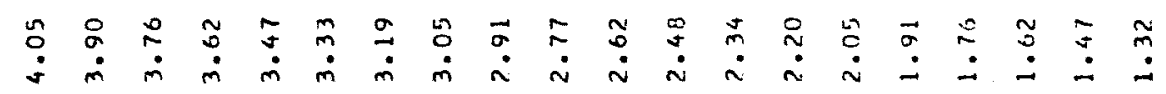

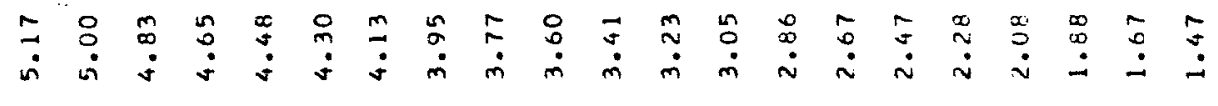

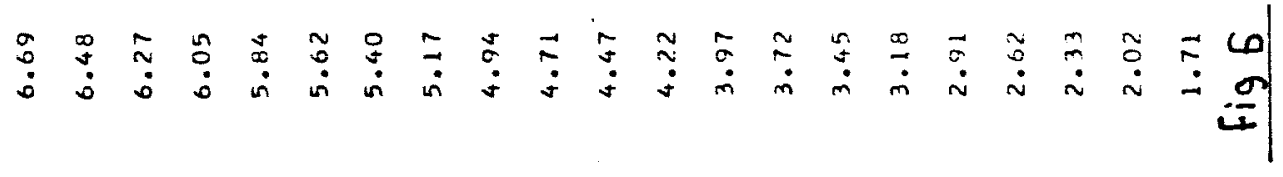

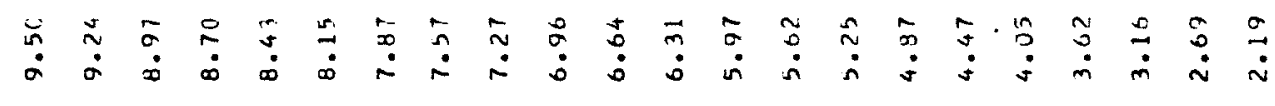

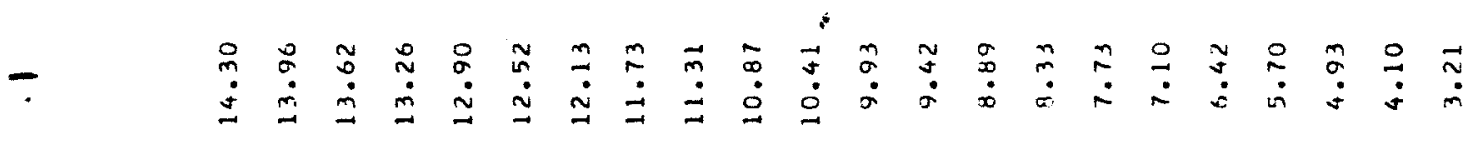

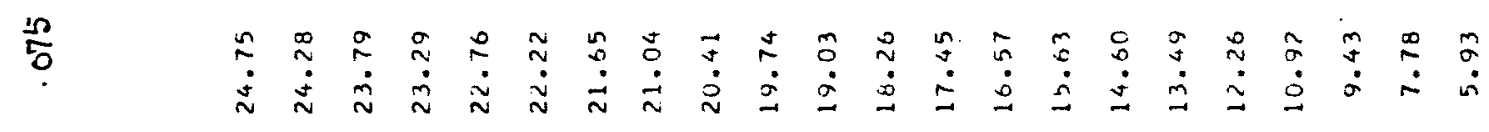

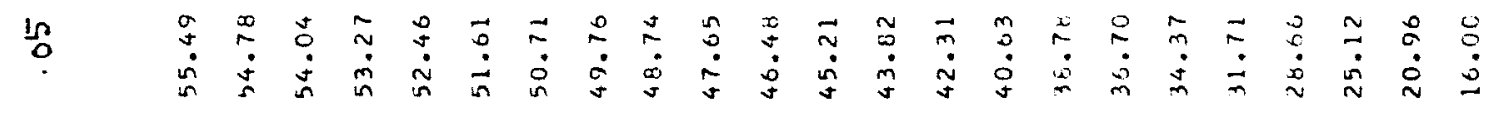

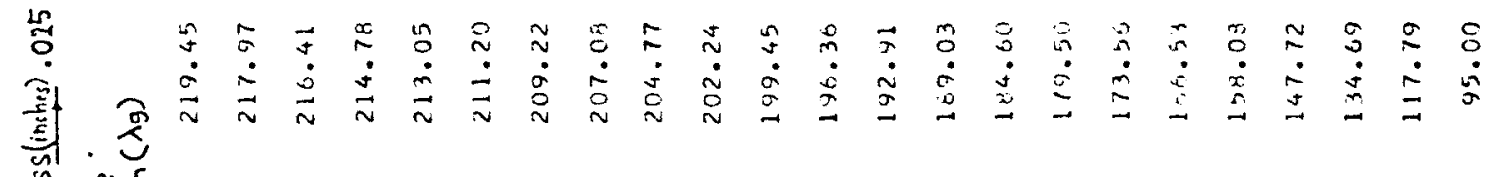

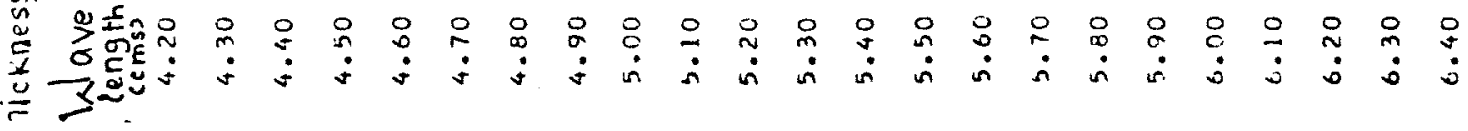




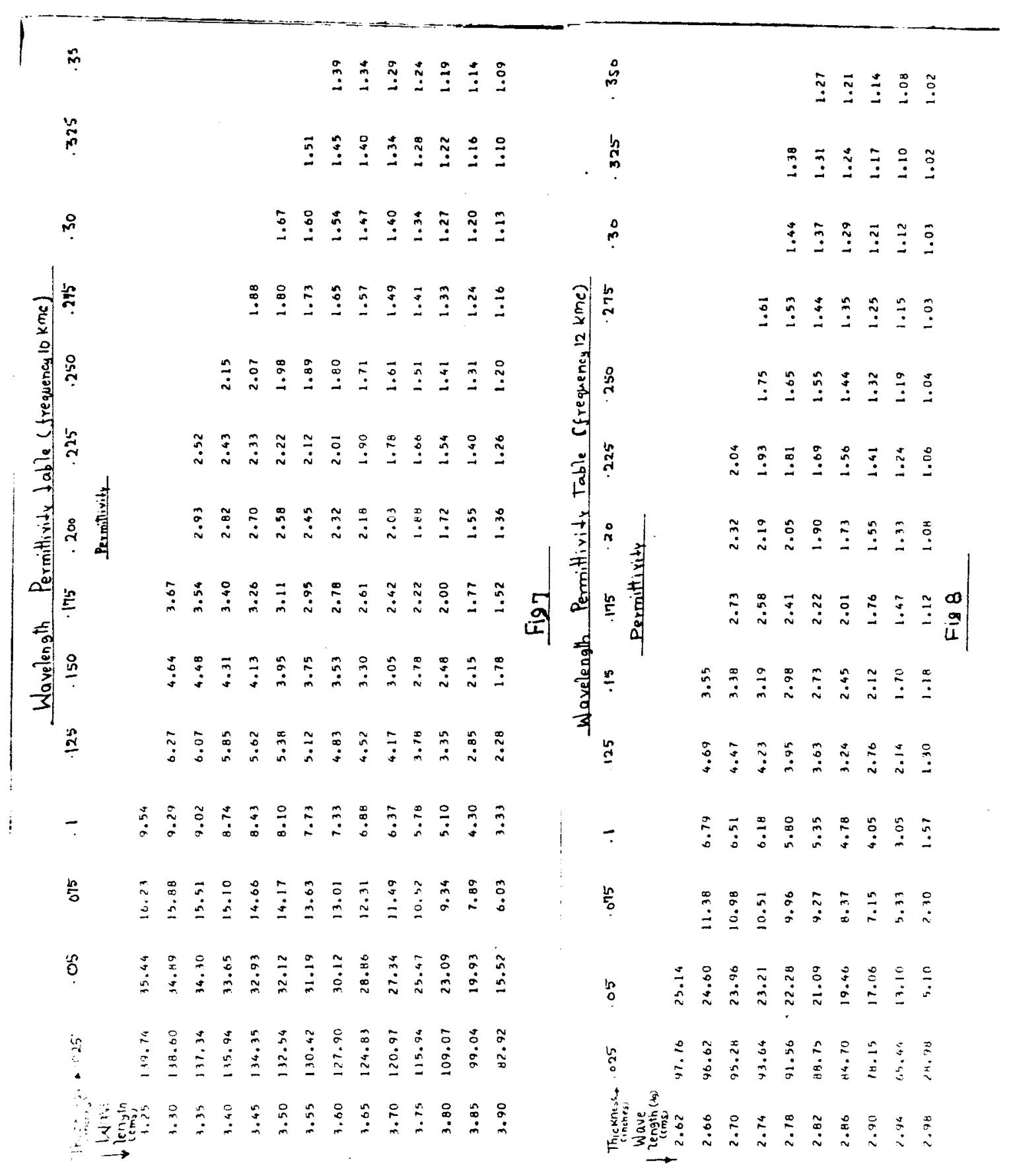




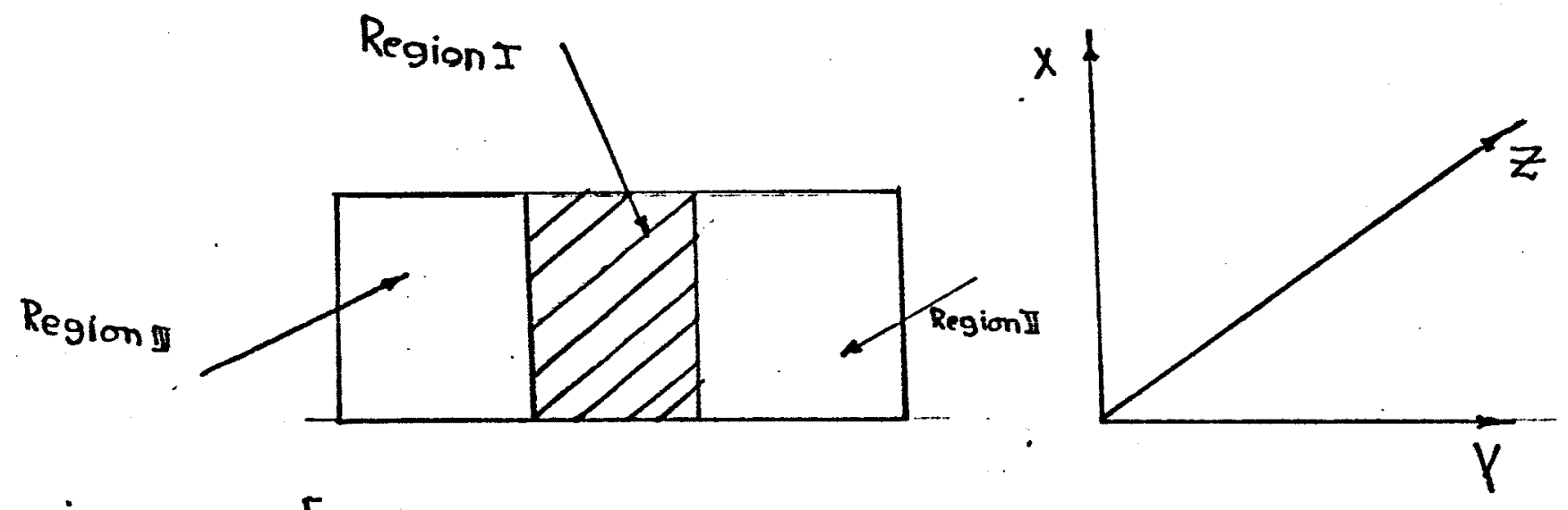

Fig 9 ) -Cross section of the cavity system when the dielectric is placed along the center of the cross section 


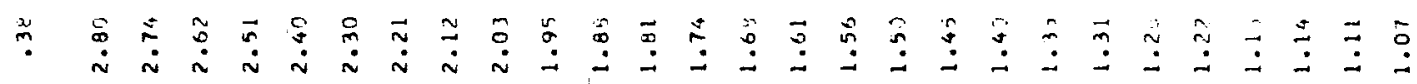

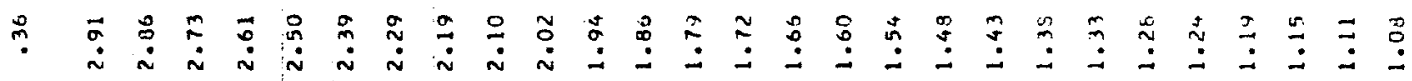

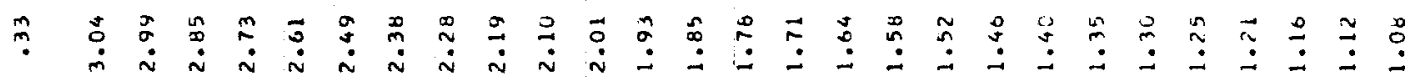

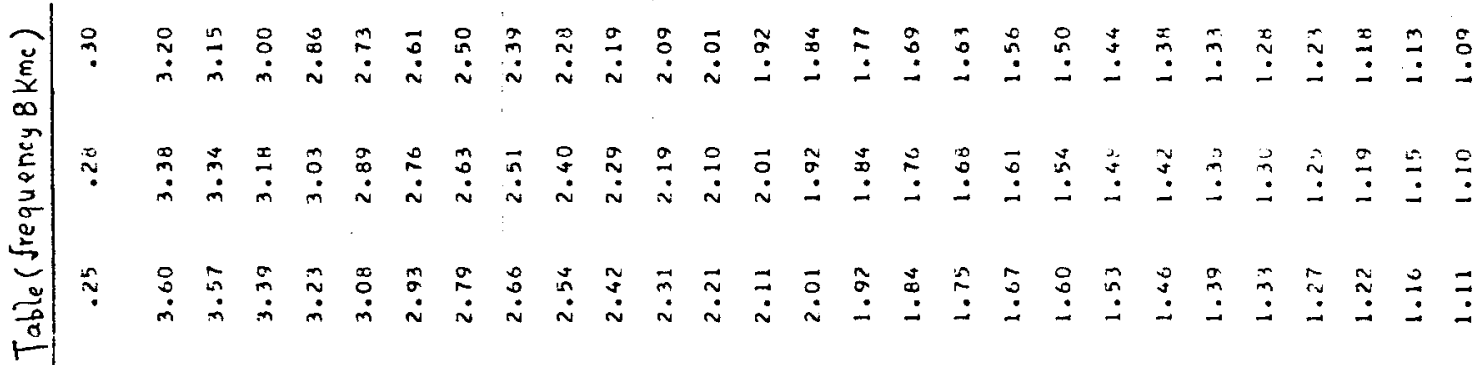

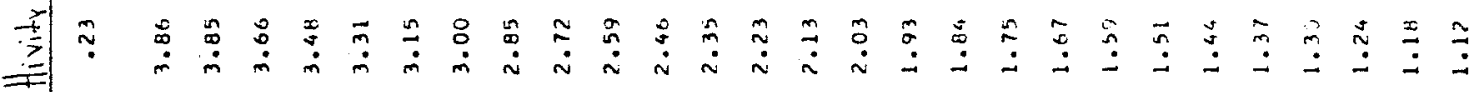

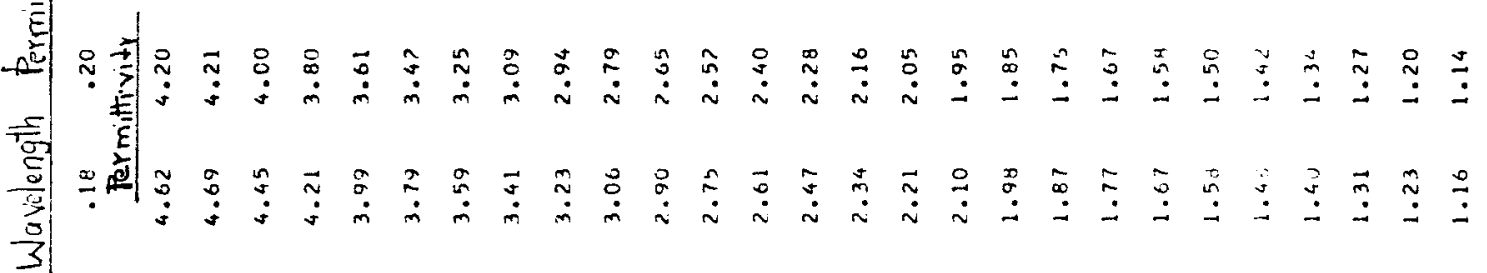

?

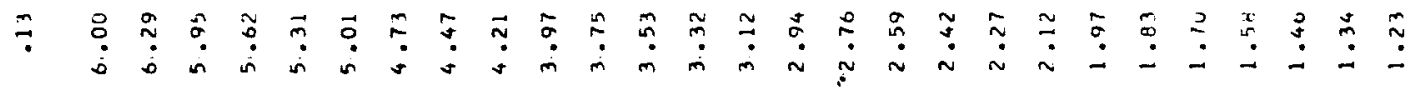

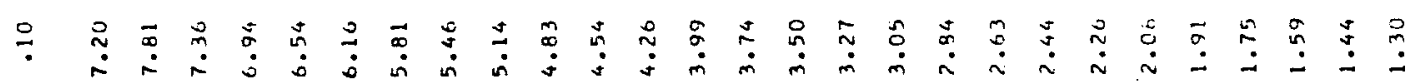

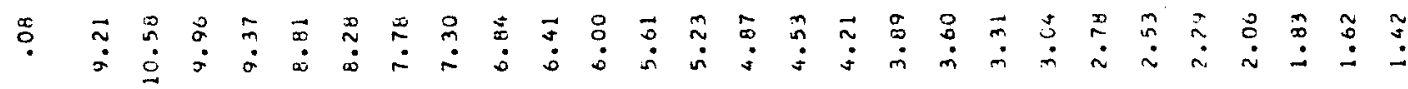

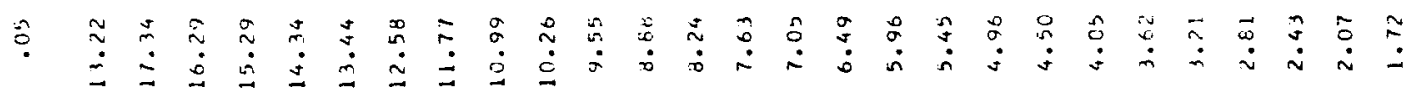

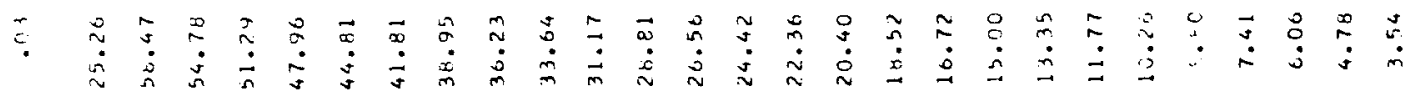

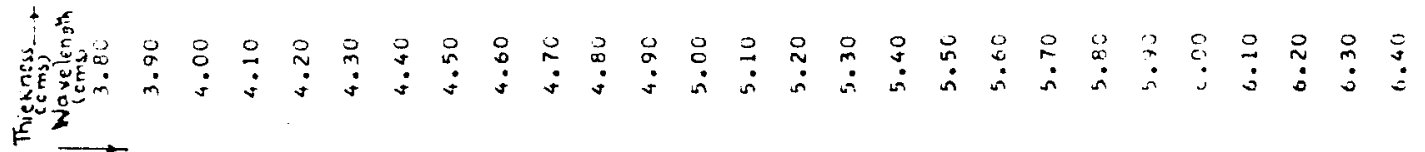




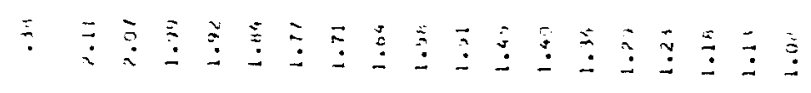

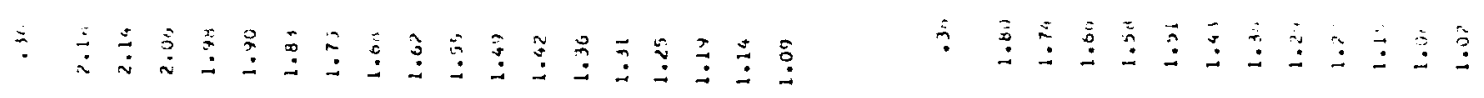

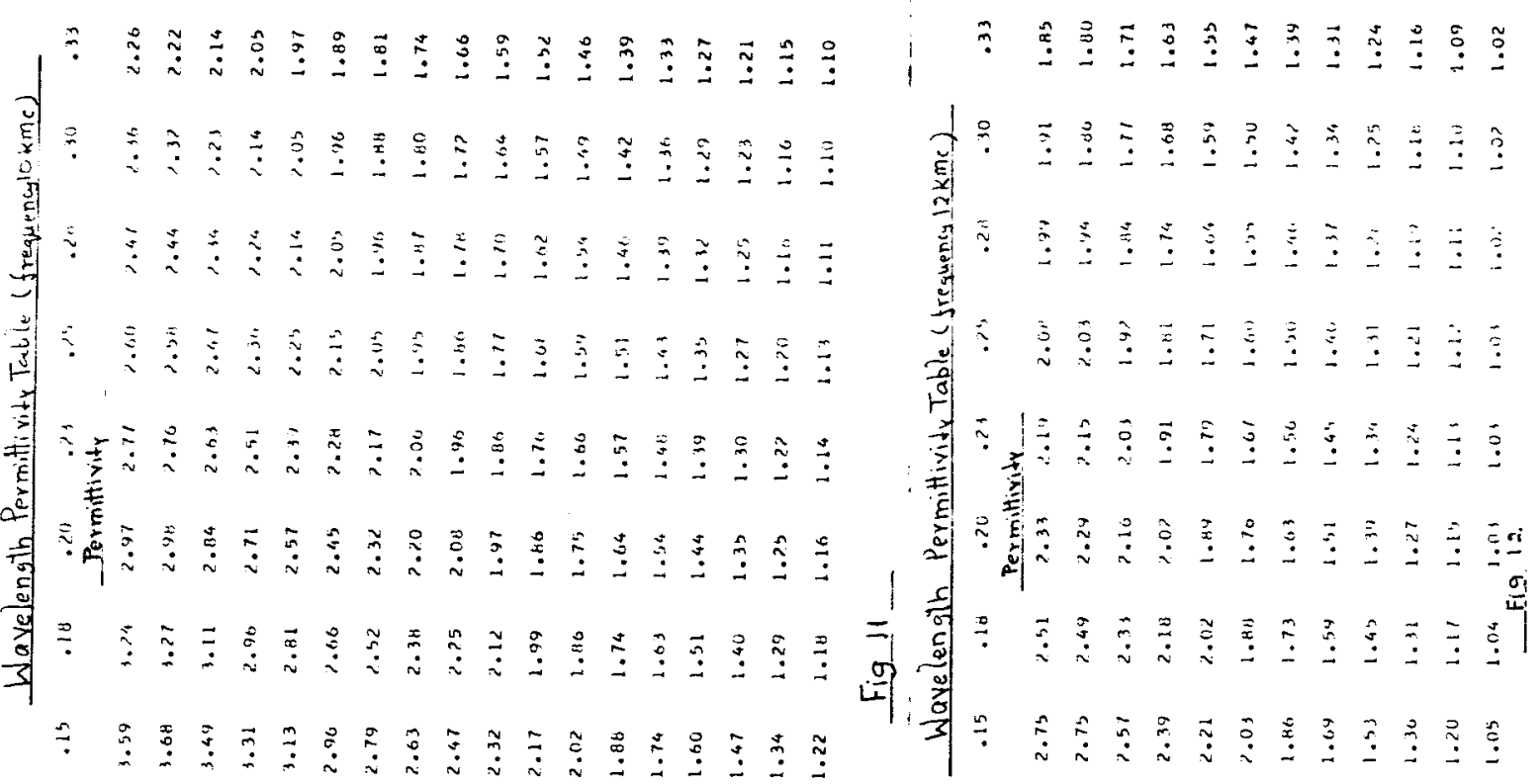

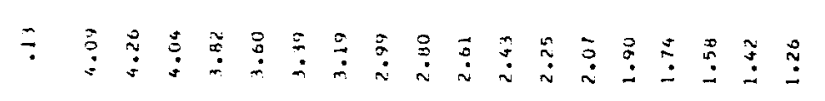

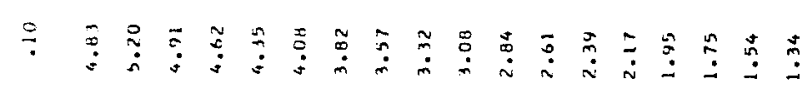

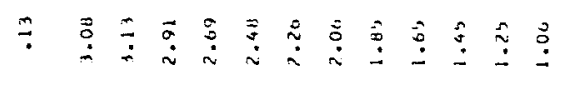

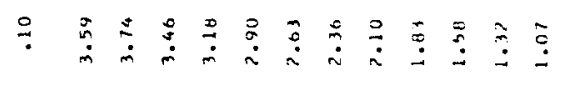

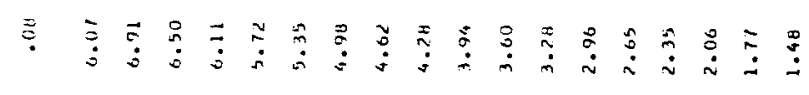

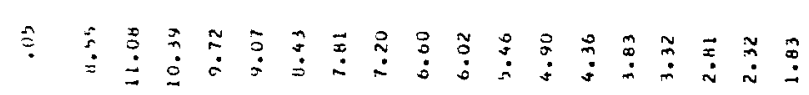

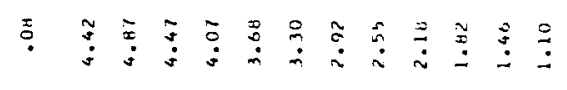
ใน

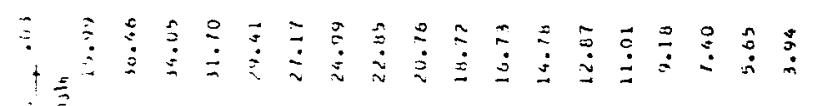

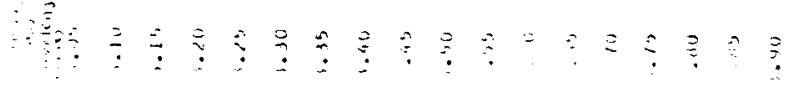

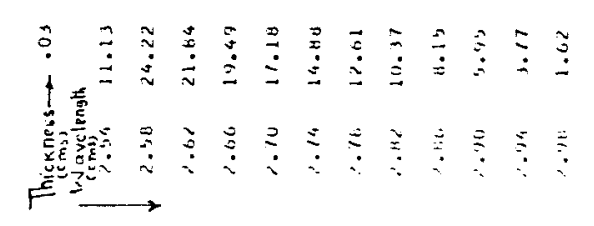




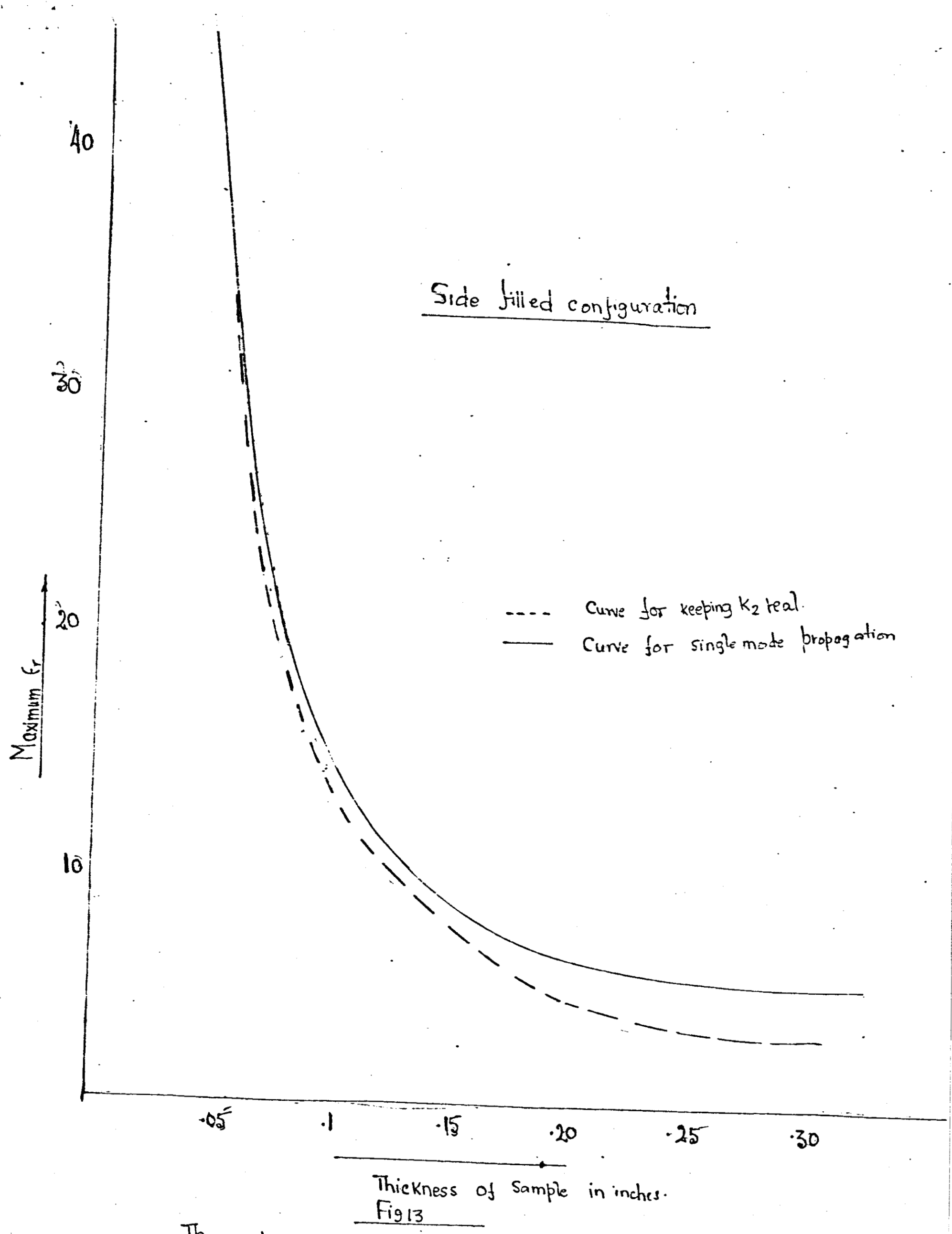

Thickness of sample in inches.

Theoretical curves correl action $\epsilon_{r}$ (max) with same? "nicknisises for the single mole brobasation and limit to the use of real functions. 

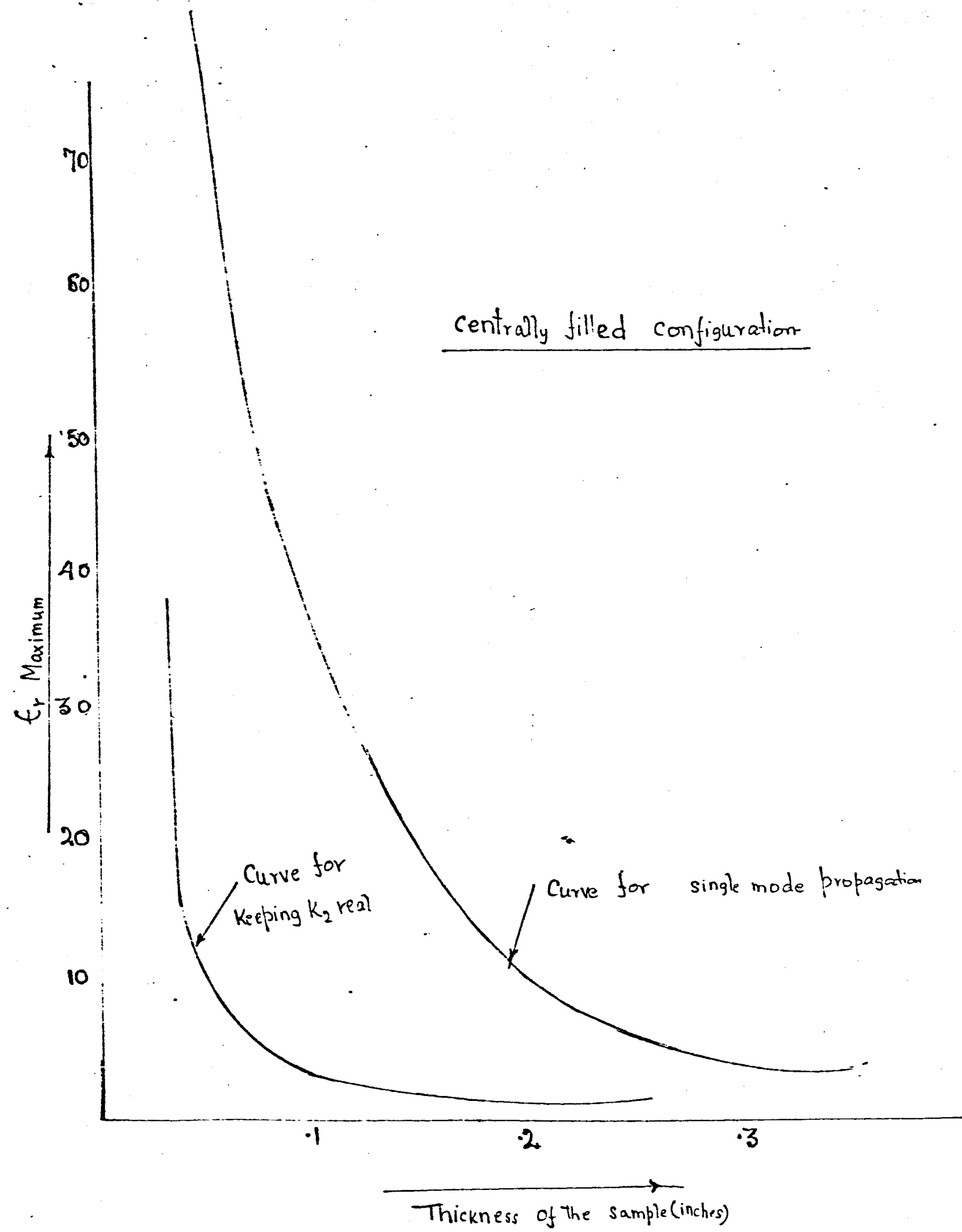

Fig 14

Theoretic a' curves correlating $t_{r}$ (max) with sample thicknesses for single mode propagation and for limit to the use of only real functions. 


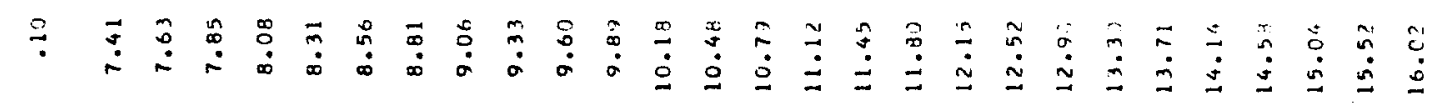

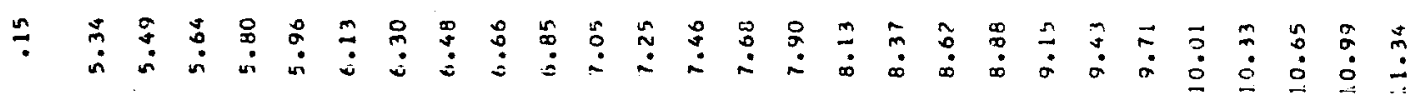

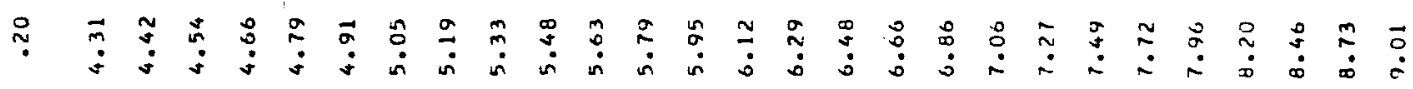

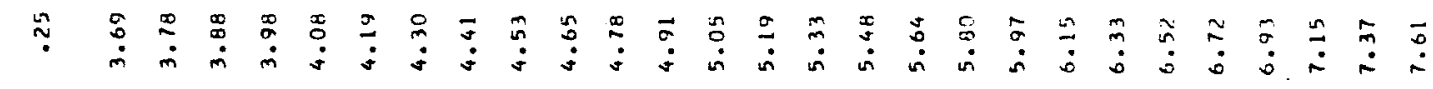

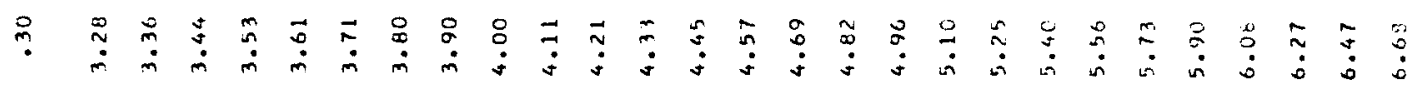

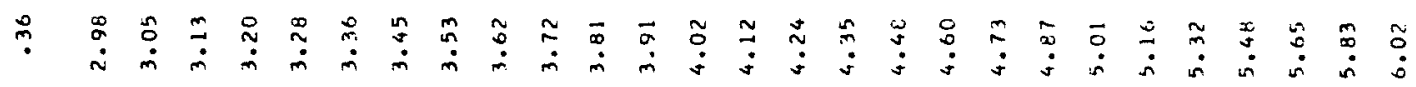

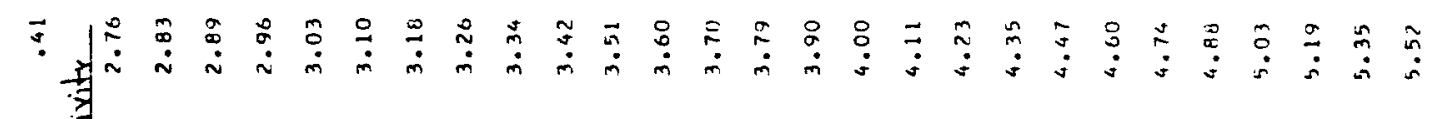

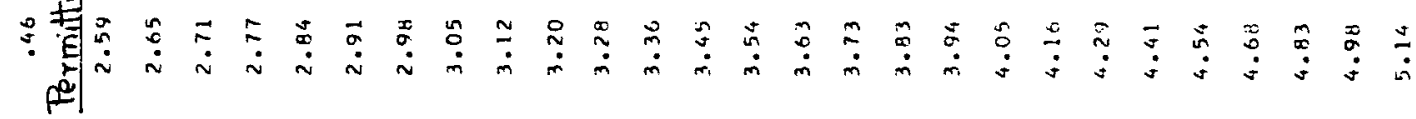

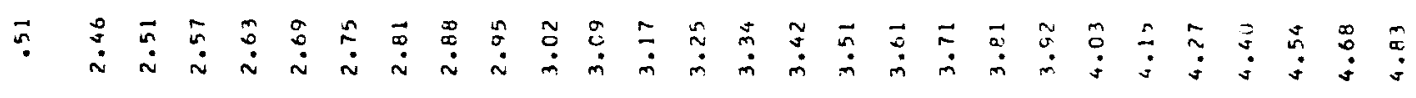

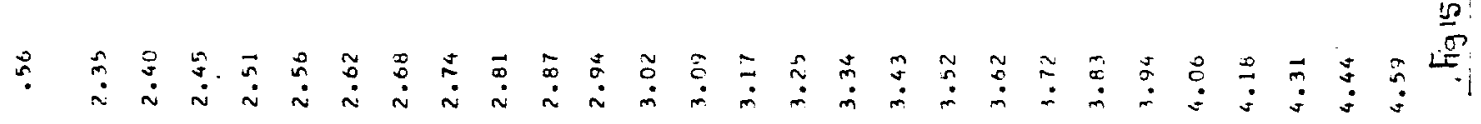

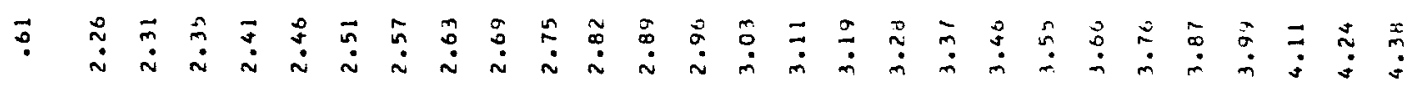

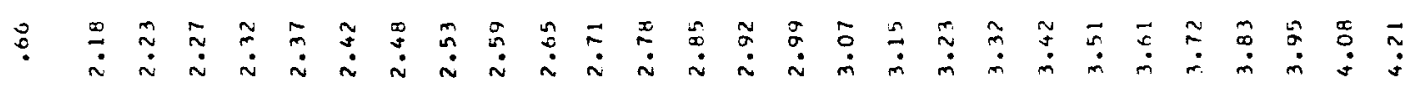

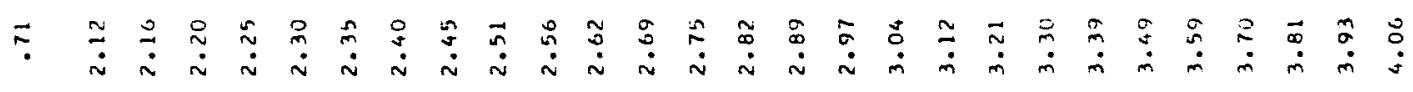

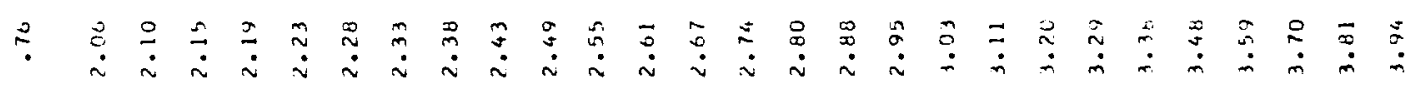

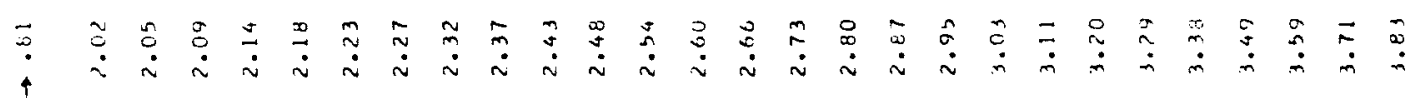

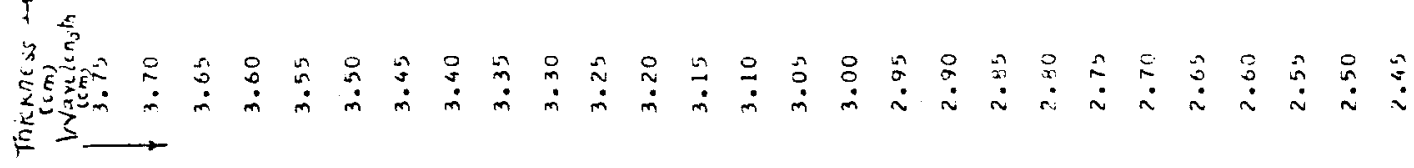




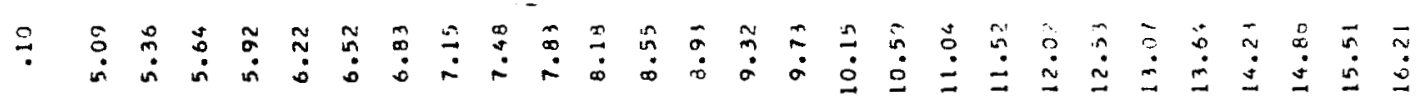

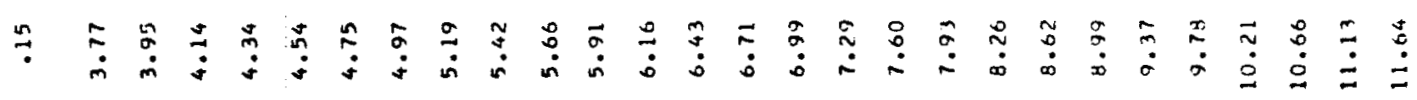

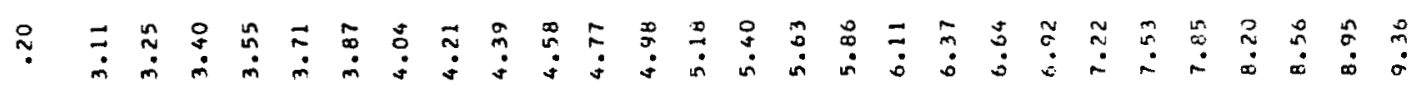

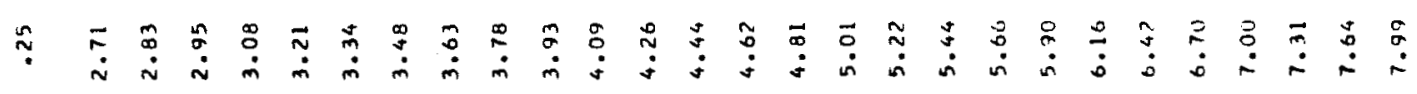

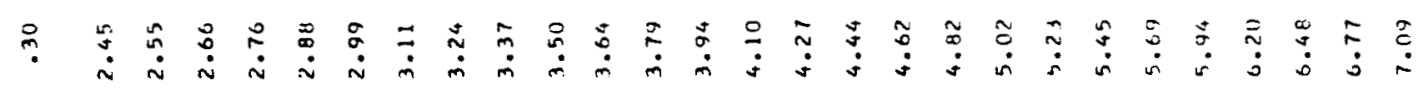

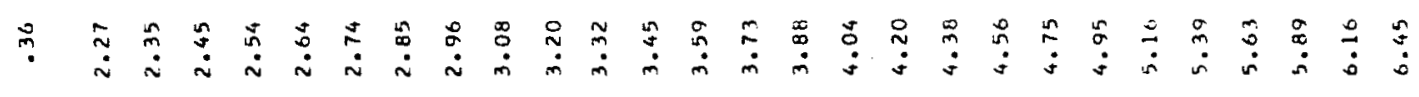

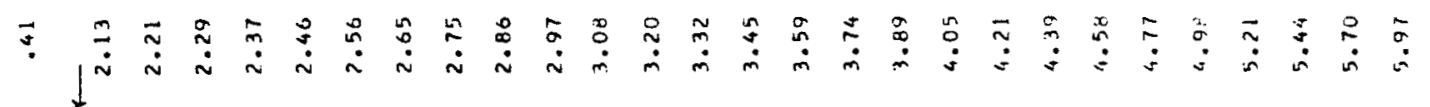

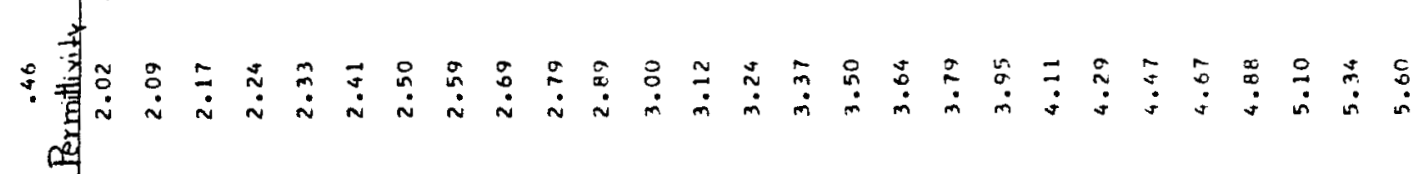

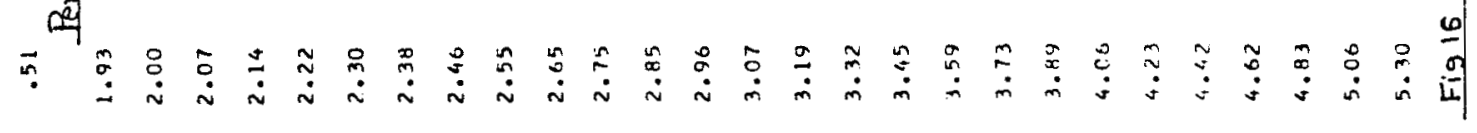

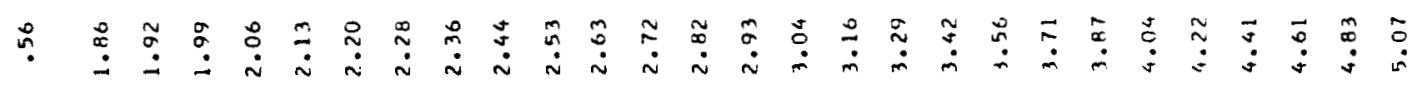

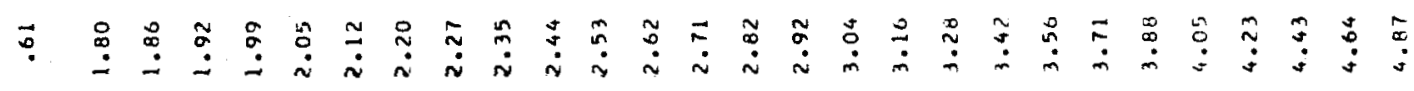

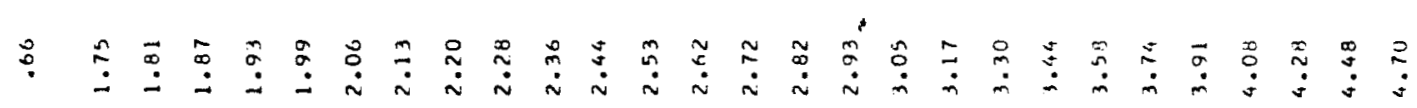

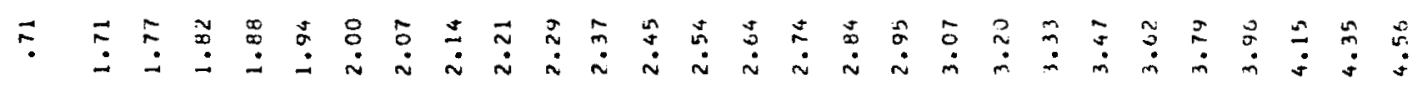

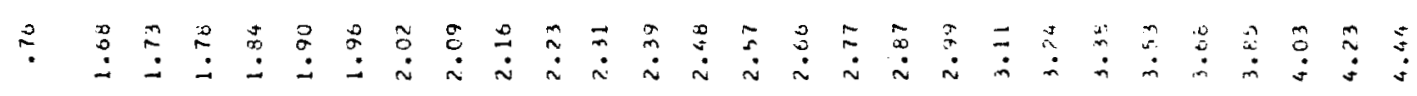

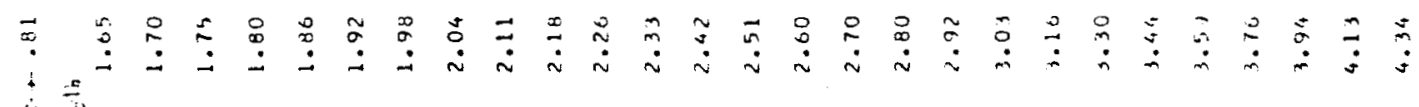

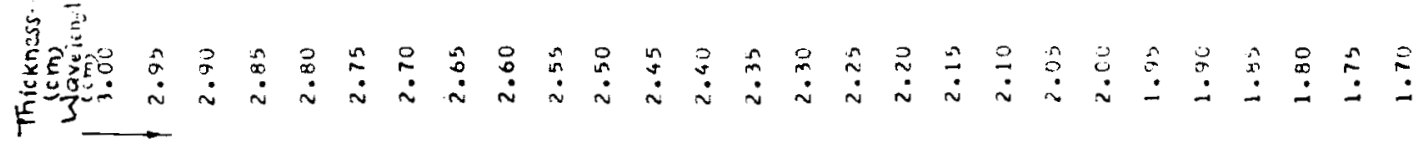




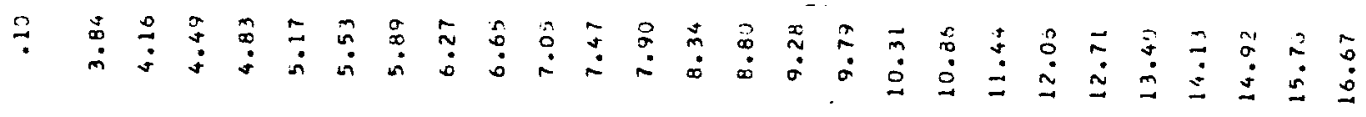

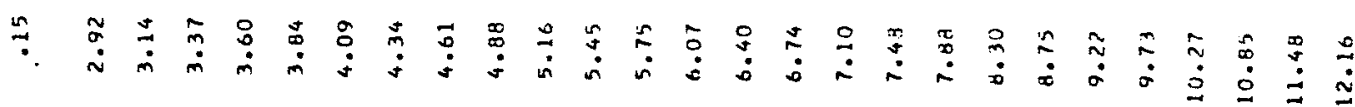

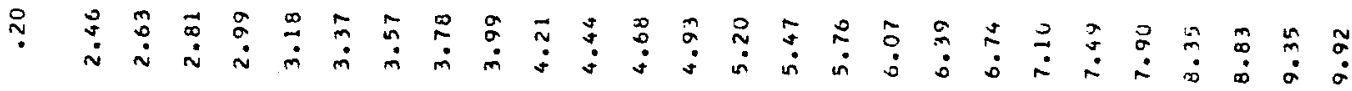

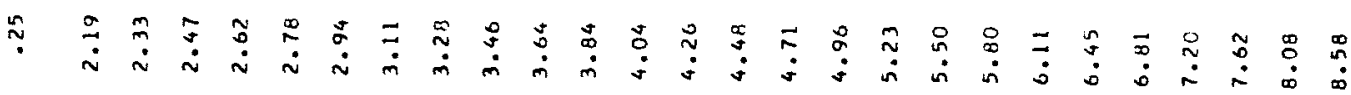

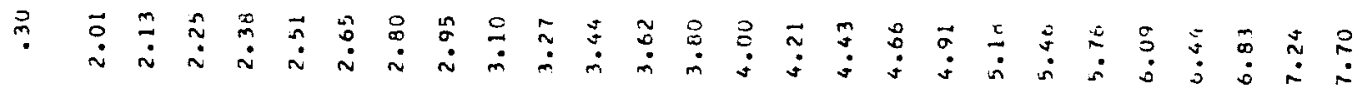

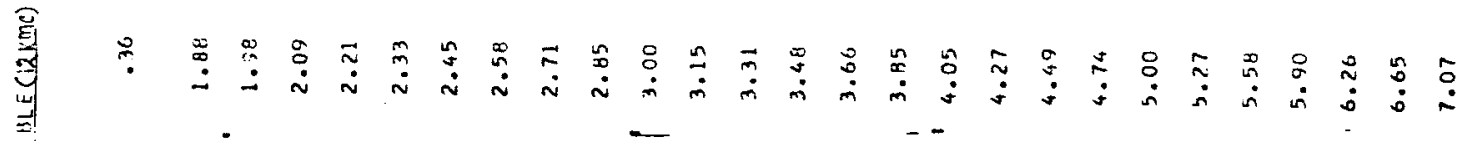

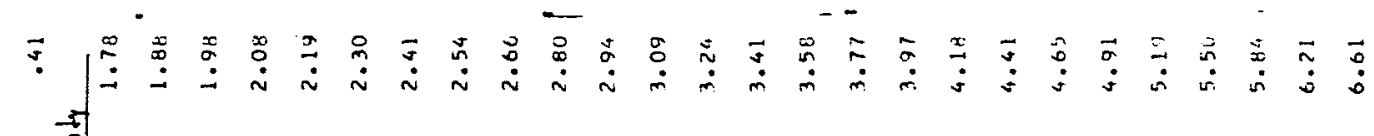

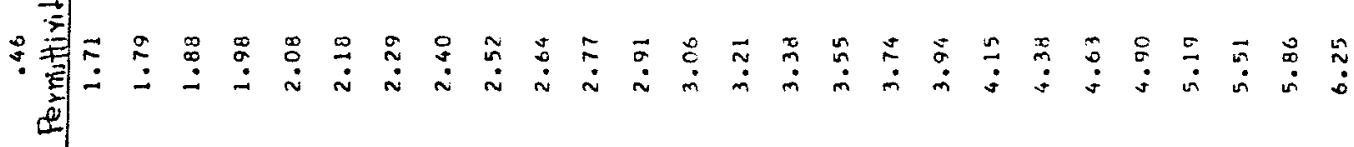

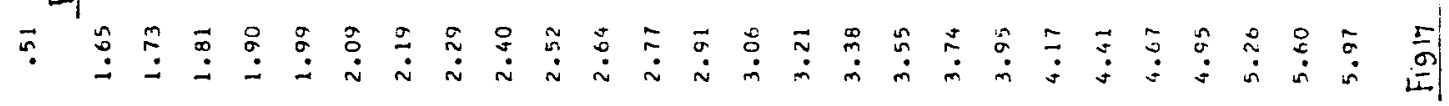

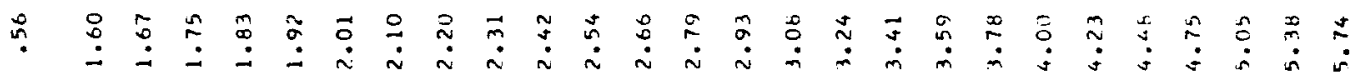

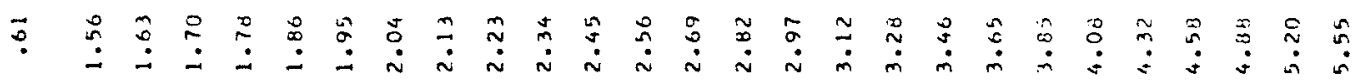

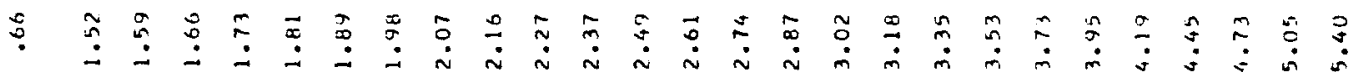

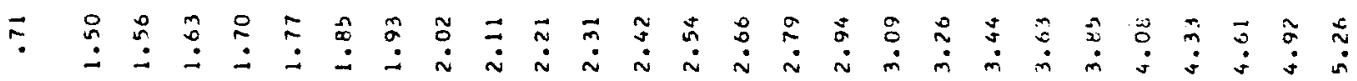

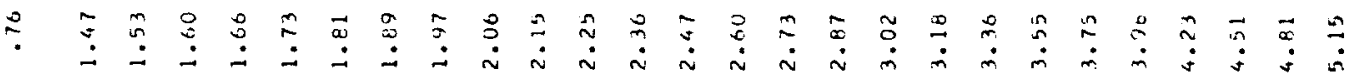

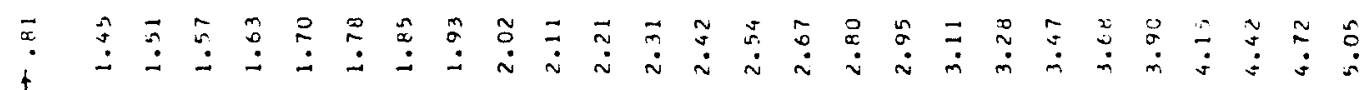

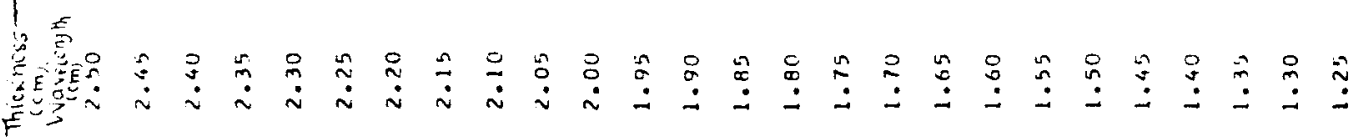




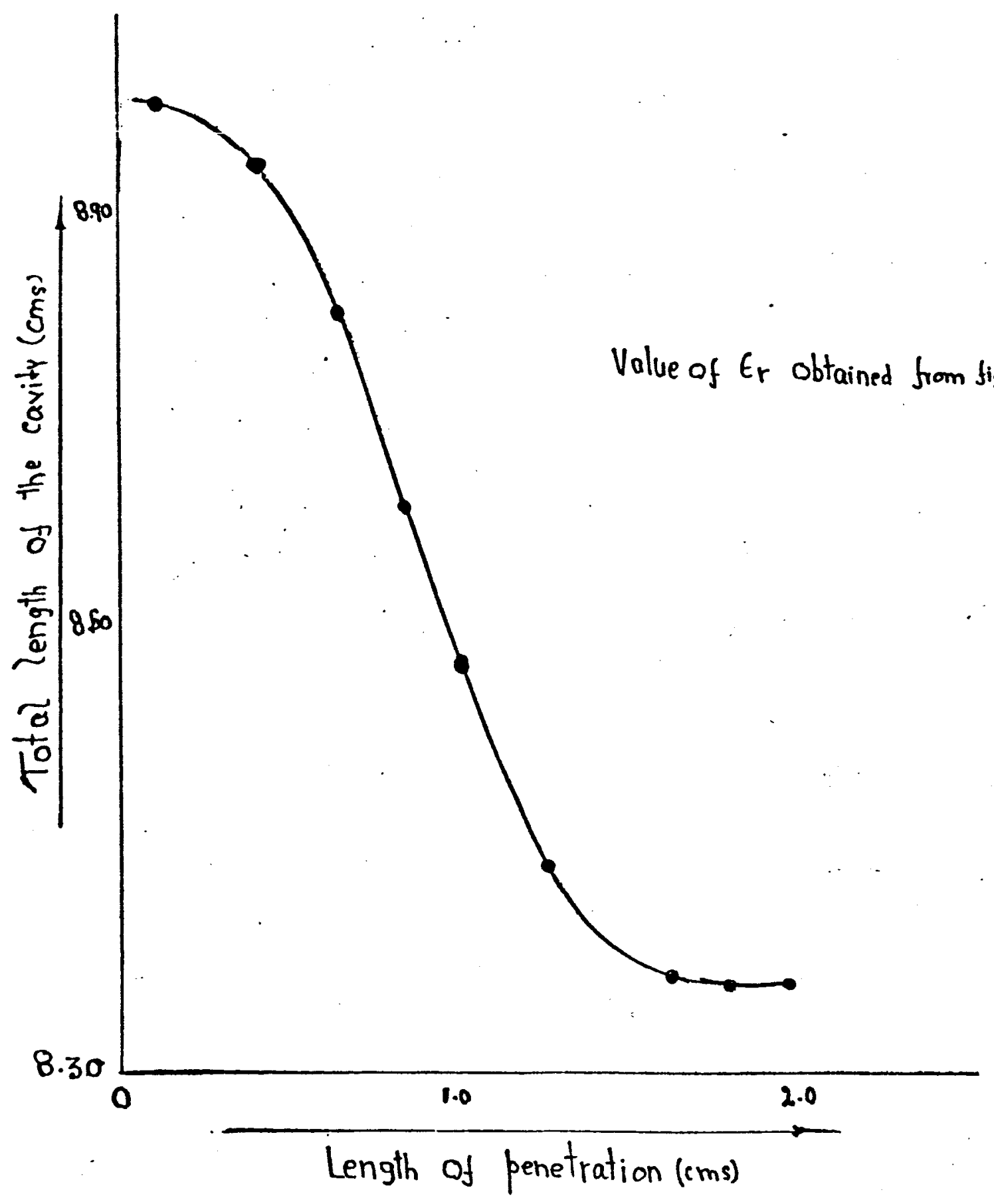

Fig fol- variation of the total length of the cavity with dielectric insertion for the side filled configuration $\left(t=-3^{4}\right)$ 


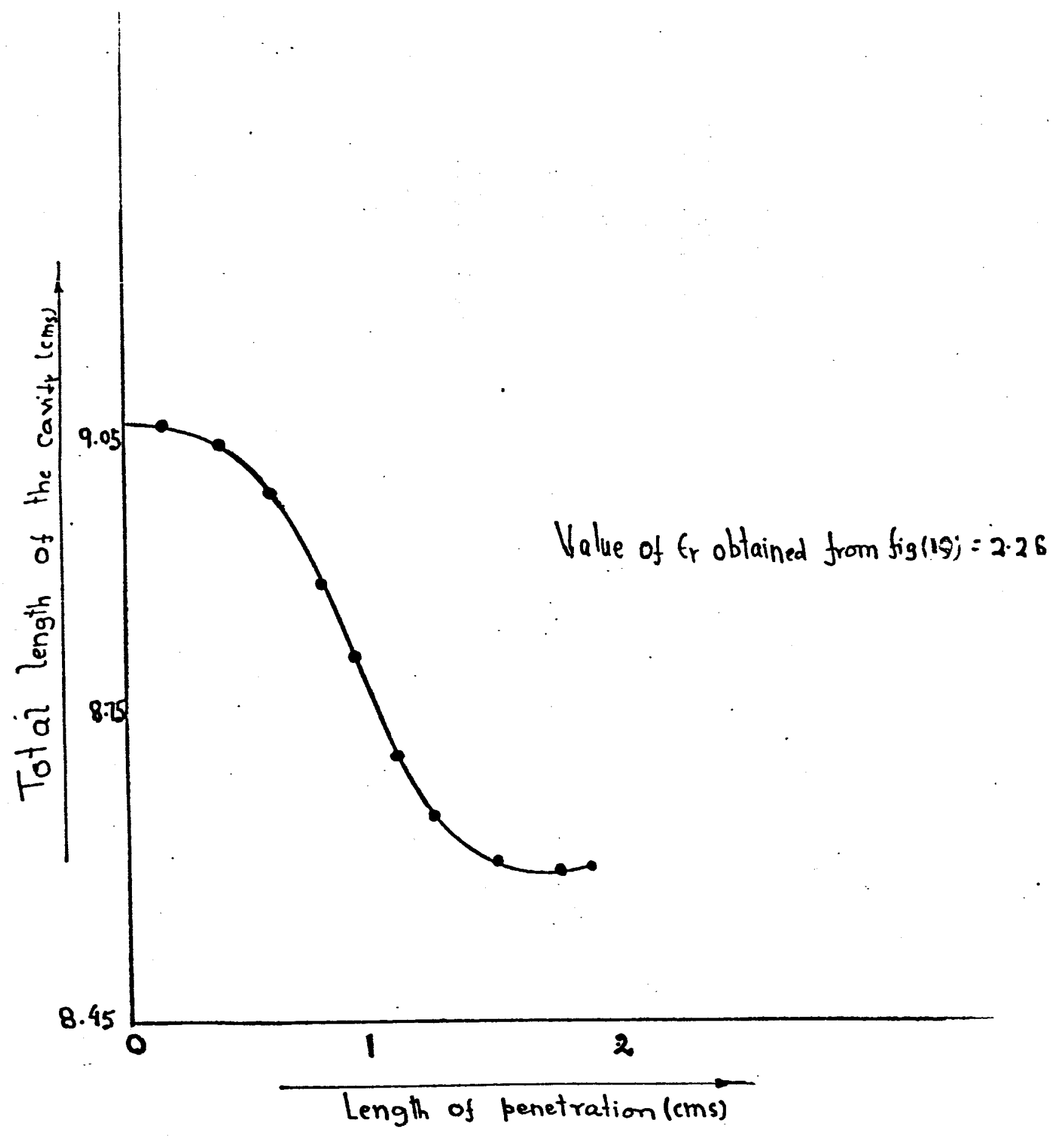

Fig is- Variation of total length of The cavity with dielectric insertion for the centrally filled configuration $t^{\prime}=1^{\prime \prime}$. 
$\because \cdot$

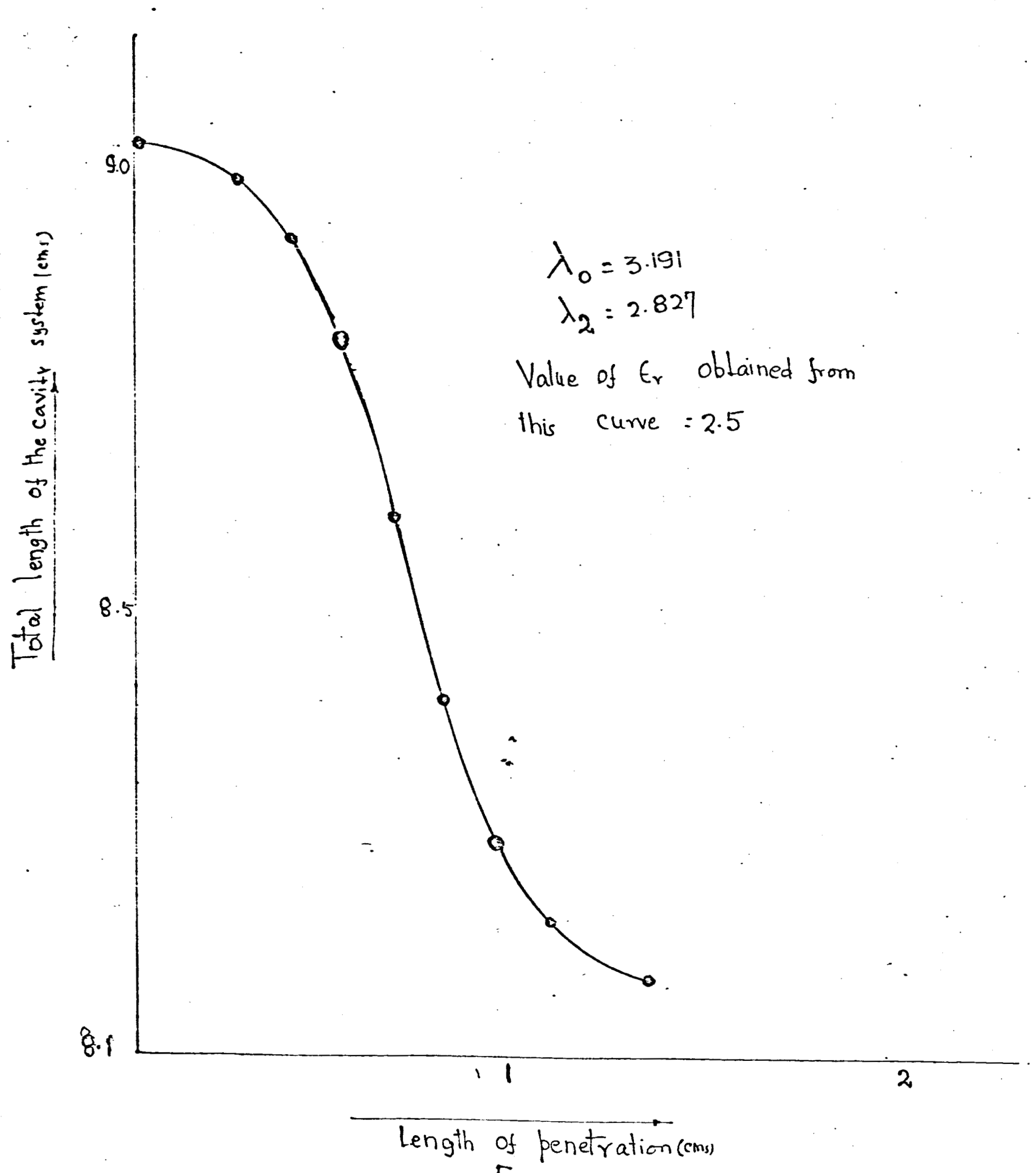

Fig (20)

Variation of total length of the cavity with dielectric insertion for centrally loaded configuration for a sample of plexiglass $\left(t^{\prime}=195^{\prime \prime}\right)$. 
